\title{
TWISTED GENERA OF SYMMETRIC PRODUCTS
}

\author{
LAURENTIU MAXIM AND JÖRG SCHÜRMANN
}

\begin{abstract}
We prove very general formulae for the generating series of (Hodge) genera of symmetric products $X^{(n)}$ with coefficients, which hold for complex quasi-projective varieties $X$ with any kind of singularities, and which include many of the classical results in the literature as special cases. Important specializations of our results include generating series for extensions of Hodge numbers and Hirzebruch's $\chi_{y}$-genus to the singular setting and, in particular, generating series for Intersection cohomology Hodge numbers and Goresky-MacPherson Intersection cohomology signatures of symmetric products of complex projective varieties. A very general proof is given based on Künneth formulae and pre-lambda structures on the coefficient theory of a point, $\bar{K}_{0}(A(p t))$, with $A(p t)$ a Karoubian $\mathbb{Q}$-linear tensor category. Moreover, Atiyah's approach to power operations in $K$-theory also works in this context for $\bar{K}_{0}(A(p t))$, giving a nice description of the important related Adams operations. This last approach also allows us to introduce very interesting coefficients on the symmetric products $X^{(n)}$.
\end{abstract}

\section{Contents}

1. Introduction

2. Symmetric products and pre-lambda rings

2.1. Pre-lambda rings

2.2. Adams operations for symmetric products

3. Equivariant genera and trace homomorphisms

4. Appendix A: Exterior products and equivariant objects 27

4.1. Cofibered Categories 27

4.2. Equivariant objects 29

4.3. Künneth morphisms 30

4.4. Examples 32

5. Appendix B: Mixed Hodge modules on symmetric products 34

References 39

Date: March 6, 2019.

2000 Mathematics Subject Classification. Primary 55S15, 20C30, 32S35, 32S60, 19L20; Secondary $14 \mathrm{C} 30$.

Key words and phrases. symmetric product, exterior product, symmetric monoidal category, generating series, genus, Hodge numbers, lambda-ring, Adams operation. 


\section{INTRODUCTION}

Some of the most interesting examples of global orbifolds are the symmetric products $X^{(n)}, n \geq 0$, of a smooth complex algebraic variety $X$. The $n$-fold symmetric product of $X$ is defined as $X^{(n)}:=X^{n} / \Sigma_{n}$, i.e., the quotient of the product of $n$ copies of $X$ by the natural action of the symmetric group on $n$ elements, $\Sigma_{n}$. If $X$ is a smooth projective curve, symmetric products are of fundamental importance in the study of the Jacobian variety of $X$ and other aspects of its geometry, e.g., see [28]. If $X$ is a smooth algebraic surface, $X^{(n)}$ is used to understand the topology of the $n$-th Hilbert scheme $X^{[n]}$ parametrizing closed zero-dimensional subschemes of length $n$ of $X$, e.g., see [20, 12]. For the purpose of this note we shall assume that $X$ is a (possibly singular) complex quasi-projective variety, therefore its symmetric products are algebraic varieties as well.

A generating series for a given invariant $\mathcal{I}(-)$ of symmetric products of complex algebraic varieties is an expression of the form

$$
\sum_{n \geq 0} \mathcal{I}\left(X^{(n)}\right) \cdot t^{n}
$$

provided $\mathcal{I}\left(X^{(n)}\right)$ can be defined for all $n$. The aim is to calculate such an expression solely in terms of invariants of $X$. Then the corresponding invariant of the $n$-th symmetric product $X^{(n)}$ is equal to the coefficient of $t^{n}$ in the resulting expression in invariants of $X$.

For example, there is a well-known formula due to Macdonald [29] for the generating series of the Betti numbers $b_{k}(X):=\operatorname{dim}\left(H^{k}(X, \mathbb{Q})\right)$ and resp. topological Euler characteristic $\chi(X):=\sum_{k \geq 0}(-1)^{k} \cdot b_{k}(X)$ of a compact triangulated space $X$ :

$$
\sum_{n \geq 0}\left(\sum_{k \geq 0} b_{k}\left(X^{(n)}\right) \cdot(-z)^{k}\right) \cdot t^{n}=\prod_{k \geq 0}\left(\frac{1}{1-z^{k} t}\right)^{(-1)^{k} \cdot b_{k}(X)}
$$

and, respectively,

$$
\sum_{n \geq 0} \chi\left(X^{(n)}\right) \cdot t^{n}=(1-t)^{-\chi(X)}=\exp \left(\sum_{r \geq 1} \chi(X) \cdot \frac{t^{r}}{r}\right) .
$$

For the last equality recall that $-\log (1-t)=\sum_{r \geq 1} \frac{t^{r}}{r}$.

A Chern class version of the formula (2) was obtained by Ohmoto in [34] for the ChernMacPherson classes of [30. Moonen [33] obtained generating series for the arithmetic genus $\chi_{a}(X):=\sum_{k \geq 0}(-1)^{k} \cdot \operatorname{dim}\left(H^{k}\left(X, \mathcal{O}_{X}\right)\right)$ of symmetric products of a complex projective variety:

$$
\sum_{n \geq 0} \chi_{a}\left(X^{(n)}\right) \cdot t^{n}=(1-t)^{-\chi_{a}(X)}=\exp \left(\sum_{r \geq 1} \chi_{a}(X) \cdot \frac{t^{r}}{r}\right)
$$


and, more generally, for the Baum-Fulton-MacPherson homology Todd classes (cf. 4]) of symmetric products of projective varieties. In [42], Zagier obtained such generating series for the signature $\sigma(X)$ and resp. L-classes of symmetric products of compact triangulated (rational homology) manifolds, e.g., for a complex projective homology manifold $X$ of pure even complex dimension he showed that

$$
\sum_{n \geq 0} \sigma\left(X^{(n)}\right) \cdot t^{n}=\frac{(1+t)^{\frac{\sigma(X)-\chi(X)}{2}}}{(1-t)^{\frac{\sigma(X)+\chi(X)}{2}}} .
$$

Borisov and Libgober obtained in [9] generating series for the Hirzebruch $\chi_{y}$-genus and, more generally, for elliptic genera of symmetric products of smooth compact varieties (cf. also [43] for other calculations involving the Hirzebruch $\chi_{y^{-}}$genus). Generating series for the Hodge numbers $h_{c}^{p, q, k}(X):=h^{p, q}\left(H_{c}^{k}(X, \mathbb{Q})\right)$ and resp. the E-polynomial

$$
e_{c}(X):=\sum_{p, q \geq 0} e_{c}^{p, q}(X) \cdot y^{p} x^{q}, \quad \text { with } \quad e_{c}^{p, q}(X):=\sum_{k \geq 0}(-1)^{k} \cdot h_{c}^{p, q, k}(X)
$$

of the cohomology with compact support of a quasi-projective variety $X$ (endowed with Deligne's mixed Hodge structure [13]) are stated by Cheah in [12]:

$$
\sum_{n \geq 0}\left(\sum_{p, q, k \geq 0} h_{c}^{p, q, k}\left(X^{(n)}\right) \cdot y^{p} x^{q}(-z)^{k}\right) \cdot t^{n}=\prod_{p, q, k \geq 0}\left(\frac{1}{1-y^{p} x^{q} z^{k} t}\right)^{(-1)^{k} \cdot h_{c}^{p, q, k}(X)}
$$

and, respectively,

$$
\sum_{n \geq 0} e_{c}\left(X^{(n)}\right) \cdot t^{n}=\prod_{p, q \geq 0}\left(\frac{1}{1-y^{p} x^{q} t}\right)^{e_{c}^{p, q}(X)}=\exp \left(\sum_{r \geq 1} e_{c}(X)\left(y^{r}, x^{r}\right) \cdot \frac{t^{r}}{r}\right) .
$$

Note that for $X$ a projective variety one has

$$
b_{k}(X)=\sum_{p, q} h_{c}^{p, q, k}(X) \quad \text { and } \quad \chi(X)=e_{c}(X)(1,1)
$$

so that one gets back (10) and (2) by putting $(y, x)=(1,1)$ in (5) and (6) Similarly, using the relation

$$
\chi_{-y}(X)=e_{c}(X)(y, 1)=: \sum_{p \geq 0} f^{p} \cdot y^{p}
$$

for a projective manifold $X$, one gets in this case for the Hirzebruch $\chi_{y}$-genus the identities:

$$
\sum_{n \geq 0} \chi_{-y}\left(X^{(n)}\right) \cdot t^{n}=\prod_{p \geq 0}\left(\frac{1}{1-y^{p} t}\right)^{f^{p}(X)}=\exp \left(\sum_{r \geq 1} \chi_{-y^{r}}(X) \cdot \frac{t^{r}}{r}\right) .
$$

Note that for $X$ a complex projective manifold one also has

$$
\chi_{a}(X)=\chi_{0}(X)=e_{c}(X)(0,1) \text { and } \sigma(X)=\chi_{1}(X)=e_{c}(X)(-1,1),
$$


so that one gets back (3) and (44) for this case by putting $(y, x)=(0,1)$ and $(y, x)=$ $(-1,1)$, respectively.

Lastly, Getzler formulated such results in [15] [prop.(5.4)] already in the general context of suitable "Künneth functors" with values in a "pseudo-abelian $\mathbb{Q}$-linear tensor category" (which in [15] is called a "Karoubian rring").

The purpose of this note is to prove a very general generating series formula for such genera "with coefficients", which holds for complex quasi-projective varieties with any kind of singularities, and which includes many of the above mentioned results as special cases. Important specializations of our result include, among others, generating series for extensions of Hodge numbers and Hirzebruch's $\chi_{y}$-genus to the singular setting with coefficients

$$
\mathcal{M} \in D^{b} \operatorname{MHM}(X)
$$

a complex of Saito's (algebraic) mixed Hodge modules ([35, 36, 37]) and, in particular, generating series for Intersection cohomology Hodge numbers and Goresky-MacPherson Intersection cohomology signatures ([18]) of complex projective varieties. Note that mixed Hodge module coefficients are already used in [16], but with other techniques and applications in mind. Besides the use of very general coefficients, our approach is close to [15]. The use of coefficients not only gives more general results, but is also needed for a functorial characteristic class version of some of our results in terms of the homology Hirzebruch classes of Brasselet-Schürmann-Yokura [10], which will be treated in a forthcoming paper. The corresponding characteristic class version will unify the mentioned results of [34, 33, 42] for Chern-, Todd- and $L$-classes.

In this paper the functorial view point is only used in three important special cases:

(a) Taking the exterior product $\mathcal{M}^{\bigotimes n}$ we get an object on $X^{n}$, which is equivariant with respect to the permutation action of the symmetric group $\Sigma_{n}$ on $X^{n}$.

(b) We push down the exterior product $\mathcal{M}^{\otimes n}$ by the projection $p_{n}: X^{n} \rightarrow X^{(n)}$ to the symmetric product $X^{(n)}$ to get a $\Sigma_{n}$-equivariant object on $X^{(n)}$, from which one can take the $\Sigma_{n}$-invariant sub-object

$$
\mathcal{M}^{(n)}:=\mathcal{P}\left(p_{n_{*}} \mathcal{M}^{\bigotimes n}\right)=\left(p_{n_{*}} \mathcal{M}^{\bigotimes n}\right)^{\Sigma_{n}} \in D^{b} \operatorname{MHM}\left(X^{(n)}\right)
$$

defined by the projector $\mathcal{P}=\frac{1}{n !} \sum_{\sigma \in \Sigma_{n}} \psi_{\sigma}=:(-)^{\Sigma_{n}}$, where the isomorphisms $\psi_{\sigma}: p_{n *} \mathcal{M}^{\bigotimes n} \rightarrow p_{n *} \mathcal{M}^{\bigotimes n}$ for $\sigma \in \Sigma_{n}$ are given by the action of $\Sigma_{n}$ on $p_{n *} \mathcal{M}^{\bigotimes n}$ (compare appendix A). Here we use the fact that $\Sigma_{n}$ acts trivially on $X^{(n)}$.

(c) We push down by a constant map $k$ to a point $p t$ for calculating the invariants we are interested in:

$$
k_{*} \mathcal{M}^{(n)}, k_{!} \mathcal{M}^{(n)} \in D^{b} \mathrm{MHM}(p t) \simeq D^{b}\left(\mathrm{mHs}^{p}\right)
$$


identifying by Saito's theory the abelian category $\operatorname{MHM}(p t)$ of mixed Hodge modules on a point with the abelian category $\mathrm{mHs}^{p}$ of graded polarizable $\mathbb{Q}$-mixed Hodge structures, with $H^{*}\left(p t, k_{*(!)} \mathcal{M}^{(n)}\right)=H_{(c)}^{*}\left(X^{(n)}, \mathcal{M}^{(n)}\right)$.

And these are related by a Künneth formula (compare with Remark 2.2)

$$
H_{(c)}^{*}\left(X^{(n)}, \mathcal{M}^{(n)}\right) \simeq\left(H_{(c)}^{*}\left(X^{n}, \mathcal{M}^{\otimes n}\right)\right)^{\Sigma_{n}} \simeq\left(\left(H_{(c)}^{*}(X, \mathcal{M})\right)^{\otimes n}\right)^{\Sigma_{n}}
$$

for the cohomology (with compact support). These are isomorphisms of graded groups of mixed Hodge structures.

For suitable choices of $\mathcal{M}, \mathcal{M}^{(n)}$ becomes a highly interesting object on the symmetric product $X^{(n)}$. In this paper we are only interested in genera of $\mathcal{M}^{(n)}$, i.e., invariants defined in terms of the mixed Hodge structure on $H_{(c)}^{*}\left(X^{(n)} ; \mathcal{M}^{(n)}\right)$. But for the characteristic class version it will become important to work directly on the singular space $X^{n}$.

From the isomorphisms (9) of graded groups of mixed Hodge structures we obtain quickly our first main result:

Theorem 1.1. Let $X$ be a complex quasi-projective variety and $\mathcal{M} \in D^{b} M H M(X)$ a bounded complex of mixed Hodge modules on $X$. For $p, q, k \in \mathbb{Z}$, denote by

$$
h_{(c)}^{p, q, k}(X, \mathcal{M}):=h^{p, q}\left(H_{(c)}^{k}(X, \mathcal{M})\right):=\operatorname{dim}\left(G r_{F}^{p} G r_{p+q}^{W} H_{(c)}^{k}(X, \mathcal{M})\right)
$$

the Hodge numbers, and by

$$
e_{(c)}(X, \mathcal{M}):=\sum_{p, q} e_{(c)}^{p, q}(X, \mathcal{M}) \cdot y^{p} x^{q}, \quad \text { with } \quad e_{(c)}^{p, q}(X, \mathcal{M}):=\sum_{k \geq 0}(-1)^{k} \cdot h_{(c)}^{p, q, k}(X, \mathcal{M})
$$

the E-polynomial of the cohomology (with compact support) $H_{(c)}^{*}(X, \mathcal{M})$ of $\mathcal{M}$, so that $e_{(c)}(X, \mathcal{M}) \in \mathbb{Z}\left[y^{ \pm 1}, x^{ \pm 1}\right]$. Recall that

$$
\mathcal{M}^{(n)}:=\left(p_{n_{*}} \mathcal{M}^{\bigotimes n}\right)^{\Sigma_{n}} \in D^{b} \operatorname{MHM}\left(X^{(n)}\right)
$$

is the $n$-th symmetric product of $\mathcal{M}$. Then the following formulae hold for the generating series of these invariants:

$$
\sum_{n \geq 0}\left(\sum_{p, q, k} h_{(c)}^{p, q, k}\left(X^{(n)}, \mathcal{M}^{(n)}\right) \cdot y^{p} x^{q}(-z)^{k}\right) \cdot t^{n}=\prod_{p, q, k}\left(\frac{1}{1-y^{p} x^{q} z^{k} t}\right)^{(-1)^{k} \cdot h_{(c)}^{p, q, k}(X, \mathcal{M})}
$$

and

$$
\begin{aligned}
\sum_{n \geq 0} e_{(c)}\left(X^{(n)}, \mathcal{M}^{(n)}\right) \cdot t^{n} & =\prod_{p, q}\left(\frac{1}{1-y^{p} x^{q} t}\right)^{e_{(c)}^{p, q}(X, \mathcal{M})} \\
& =\exp \left(\sum_{r \geq 1} e_{(c)}(X, \mathcal{M})\left(y^{r}, x^{r}\right) \cdot \frac{t^{r}}{r}\right) .
\end{aligned}
$$


The definition of the E-polynomial and Hodge numbers $h_{(c)}^{p, q, k}(X, \mathcal{M})$ uses both the Hodge and the weight filtration of the mixed Hodge structure of $H_{(c)}^{k}(X, \mathcal{M})$, and it is known that these invariants can't be generalized to suitable characteristic classes (see [10]). For characteristic class versions one has to work only with the Hodge filtration $F$

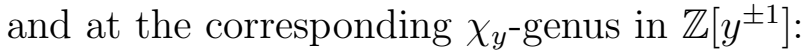

$$
\chi_{-y}^{(c)}(X, \mathcal{M}):=\sum_{p} f_{(c)}^{p} \cdot y^{p}, \quad \text { with } \quad f_{(c)}^{p}:=\sum_{i}(-1)^{i} \operatorname{dim}_{\mathbb{C}} \operatorname{Gr}_{F}^{p} H_{(c)}^{i}(X, \mathcal{M}) .
$$

Then $f_{(c)}^{p}=\sum_{q} e_{(c)}^{p, q}$ and $\chi_{-y}^{(c)}(X, \mathcal{M})=e_{(c)}(X, \mathcal{M})(y, 1)$, so Theorem 1.1 implies the following

Corollary 1.2. Let $X$ be a complex quasi-projective variety and $\mathcal{M} \in D^{b} M H M(X)$ a bounded complex of mixed Hodge modules on $X$. With the above notations, the following formula holds:

$$
\begin{aligned}
\sum_{n \geq 0} \chi_{-y}^{(c)}\left(X^{(n)}, \mathcal{M}^{(n)}\right) \cdot t^{n} & =\prod_{p}\left(\frac{1}{1-y^{p} t}\right)^{f_{(c)}^{p}(X, \mathcal{M})} \\
& =\exp \left(\sum_{r \geq 1} \chi_{-y^{r}}^{(c)}(X, \mathcal{M}) \cdot \frac{t^{r}}{r}\right) .
\end{aligned}
$$

This is in fact the formula which will be generalized in a forthcoming paper to a characteristic class version.

For the constant Hodge module (complex) $\mathcal{M}=\mathbb{Q}_{X}^{H}$ we get back (5) and (6) since, as we will show later (see Lemma 5.3),

$$
\left(\mathbb{Q}_{X}^{H}\right)^{(n)}=\mathbb{Q}_{X^{(n)}}^{H}
$$

and Deligne's and Saito's mixed Hodge structure on $H_{(c)}^{*}(X, \mathbb{Q})$ agree $([38])$. Here we only need this deep result for $X$ quasi-projective, where it quickly follows from the construction.

But of course we can use other coefficients, e.g. for $X$ pure dimensional we can use a corresponding (shifted) intersection cohomology mixed Hodge module

$$
I C^{\prime H}=I C_{X}^{H}\left[-\operatorname{dim}_{\mathbb{C}} X\right] \in \operatorname{MHM}(X)\left[-\operatorname{dim}_{\mathbb{C}} X\right] \subset D^{b} \operatorname{MHM}(X)
$$

calculating the intersection (co)homology $I H_{(c)}^{*}(X)=H_{(c)}^{*}\left(X, I C^{\prime}{ }_{X}\right)$ of $X$. Then we have similarly that (cf. Lemma 5.4)

$$
\left(I C_{X}^{\prime H}\right)^{(n)}=I C_{X}^{\prime(n)}
$$

More generally, if $\mathcal{L}$ is an admissible (graded polarizable) variation of mixed Hodge structures with quasi-unipotent monodromy at infinity defined on a smooth pure dimensional quasi-projective variety $U$, then this corresponds by Saito's work to a shifted 
mixed Hodge module

$$
\mathcal{L}^{H} \in \operatorname{MHM}(U)\left[-\operatorname{dim}_{\mathbb{C}} U\right] \subset D^{b} \operatorname{MHM}(U) .
$$

Note that the projection $p_{n}: U^{n} \rightarrow U^{(n)}$ is a finite ramified covering branched along the "fat diagonal", i.e., the induced map of the configuration spaces on $n$ (un)ordered points in $U$ :

$$
p_{n}: F(U, n):=\left\{\left(x_{1}, x_{2}, \ldots, x_{n}\right) \in U^{n} \mid x_{i} \neq x_{j} \text { for } i \neq j\right\} \rightarrow F(U, n) / \Sigma_{n}=: B(U, n)
$$

is a finite unramified covering. Therefore $\left(\mathcal{L}^{H}\right)^{(n)}$ is also a shifted mixed Hodge module with $\left(\mathcal{L}^{H}\right)^{(n)} \mid B(U, n)$ corresponding to an admissible variation of mixed Hodge structures on $B(U, n)$ (as before). For $U \subset X$ a Zariski open dense subset of a quasi-projective variety $X$, one can extend $\mathcal{L}^{H}$ and $\left(\mathcal{L}^{H}\right)^{(n)} \mid B(U, n)$ uniquely to twisted intersection cohomology mixed Hodge modules

$$
I C_{X}^{H}(\mathcal{L}) \in \operatorname{MHM}(X) \quad \text { and } \quad I C_{X^{(n)}}^{H}\left(\mathcal{L}^{(n)}\right) \in \operatorname{MHM}\left(X^{(n)}\right) .
$$

Again the shifted complexes

$$
I C^{\prime H}(\mathcal{L}):=I C_{X}^{H}(\mathcal{L})\left[-\operatorname{dim}_{\mathbb{C}} X\right] \quad \text { and } \quad I C^{\prime H}{ }_{X^{(n)}}^{H}\left(\mathcal{L}^{(n)}\right):=I C_{X^{(n)}}^{H}\left(\mathcal{L}^{(n)}\right)\left[-n \cdot \operatorname{dim}_{\mathbb{C}} X\right]
$$

calculate the corresponding twisted Intersection (co)homology. And these are related by (cf. Lemma 5.5)

$$
\left(I C_{X}^{\prime H}(\mathcal{L})\right)^{(n)}=I C^{\prime H}{ }^{(n)}\left(\mathcal{L}^{(n)}\right)
$$

Thus we get all the generating series formulae also for these (twisted) intersection (co)homology invariants, and in particular for

$$
I \chi_{-y}^{(c)}(X, \mathcal{L}):=\chi_{-y}^{(c)}\left(X, I C_{X}^{\prime H}(\mathcal{L})\right)
$$

And this polynomial unifies the following invariants:

$(\mathrm{y}=1) I \chi_{-1}^{(c)}(X, \mathcal{L})=\chi_{(c)}^{I H}(X, \mathcal{L})$ is the corresponding intersection (co)homology Euler characteristic (with compact support).

$(\mathrm{y}=0) I \chi_{0}^{(c)}(X, \mathcal{L})=I \chi_{a}^{(c)}(X, \mathcal{L})$ is the corresponding intersection (co)homology arithmetic genus (with compact support).

$(\mathrm{y}=-1)$ Assume finally that $X$ is projective of pure even complex dimension $2 m$, with $\mathcal{L}$ a polarizable variation of pure Hodge structures with quasi-unipotent monodromy at infinity defined on a smooth Zariski open dense subset $U \subset X$. If $\mathcal{L}$ is of even weight, then the middle intersection (co)homology group

$$
I H^{m}(X, \mathcal{L})=H^{m}\left(X, I C^{\prime}{ }_{X}(\mathcal{L})\right)
$$

gets an induced symmetric (non-degenerate) intersection form, with

$$
I \chi_{1}(X, \mathcal{L})=\sigma(X, \mathcal{L}):=\sigma\left(I H^{m}(X, \mathcal{L})\right)
$$

the corresponding Goresky-MacPherson (twisted intersection homology) signature ([18]). This identification follows from Saito's Hodge index theorem for $I H^{*}(X, \mathcal{L})([35,37])$. 
Here we only formulate the following

Corollary 1.3. Let $X$ be a pure dimensional complex quasi-projective variety, with $\mathcal{L}$ an admissible (graded polarizable) variation of mixed Hodge structures with quasi-unipotent monodromy at infinity defined on a smooth Zariski open dense subset of $X$. Then

$$
\begin{gathered}
\sum_{n \geq 0} I \chi_{-y}^{(c)}\left(X^{(n)}, \mathcal{L}^{(n)}\right) \cdot t^{n}=\exp \left(\sum_{r \geq 1} I \chi_{-y^{r}}^{(c)}(X, \mathcal{L}) \cdot \frac{t^{r}}{r}\right) \\
\sum_{n \geq 0} \chi_{(c)}^{I H}\left(X^{(n)}, \mathcal{L}^{(n)}\right) \cdot t^{n}=\exp \left(\sum_{r \geq 1} \chi_{(c)}^{I H}(X, \mathcal{L}) \cdot \frac{t^{r}}{r}\right)=(1-t)^{-\chi_{(c)}^{I H}(X, \mathcal{L})} . \\
\sum_{n \geq 0} I \chi_{a}^{(c)}\left(X^{(n)}, \mathcal{L}^{(n)}\right) \cdot t^{n}=\exp \left(\sum_{r \geq 1} I \chi_{a}^{(c)}(X, \mathcal{L}) \cdot \frac{t^{r}}{r}\right)=(1-t)^{-I \chi_{a}^{(c)}(X, \mathcal{L})} .
\end{gathered}
$$

Finally, assume in addition that $X$ is projective of even complex dimension, with $\mathcal{L}$ a polarizable variation of pure Hodge structures of even weight. Then

$$
\sum_{n \geq 0} \sigma\left(X^{(n)}, \mathcal{L}^{(n)}\right) \cdot t^{n}=\frac{(1+t)^{\frac{\sigma(X, \mathcal{L})-\chi^{I H}(X, \mathcal{L})}{2}}}{(1-t)^{\frac{\sigma(X, \mathcal{L})+\chi^{I H}(X, \mathcal{L})}{2}}}
$$

Note that the right-hand side of equation (21) is a rational function in $t$ since, by Poincaré duality in twisted intersection homology, the Goresky-MacPherson signature and the intersection homology Euler characteristic have the same parity. In the special case of a projective rational homology manifold $X$ one has $I C^{\prime H} \simeq \mathbb{Q}_{X}^{H}$ and $I H^{*}(X)=H^{*}(X)$, so that we then get back the result (4) by Hirzebruch and Zagier 42 .

Finally, our method of proof also gives the following closely related results:

\section{Theorem 1.4. Assume}

(a) $X$ is a complex algebraic variety or a compact complex analytic set (or a real semi-algebraic or compact subanalytic set, or a compact Whitney stratified set). Let $\mathcal{F} \in D_{c}^{b}(X)$ be a bounded complex of sheaves of vector spaces over a field $\mathbb{K}$ of characteristic zero, which is constructible (in the corresponding sense). Then $H_{(c)}^{*}(X, \mathcal{F})$ is finite dimensional ([39]), so that the corresponding Euler characteristic

$$
\chi_{(c)}(X, \mathcal{F}):=\chi\left(H_{(c)}^{*}(X, \mathcal{F})\right)
$$

is defined. Then the same is true for $\chi_{(c)}\left(X^{(n)}, \mathcal{F}^{(n)}\right)$, and

$$
\sum_{n \geq 0} \chi_{(c)}\left(X^{(n)}, \mathcal{F}^{(n)}\right) \cdot t^{n}=(1-t)^{-\chi_{(c)}(X, \mathcal{F})}=\exp \left(\sum_{r \geq 1} \chi_{(c)}(X, \mathcal{F}) \cdot \frac{t^{r}}{r}\right) .
$$


(b) $X$ is a compact complex algebraic variety or analytic set, and $\mathcal{F} \in D_{\text {coh }}^{b}(X)$ is a bounded complex of sheaves of $\mathcal{O}_{X}$-modules with coherent cohomology sheaves. Then $H^{*}(X, \mathcal{F})$ is finite dimensional by Serre's finiteness theorem. So one can define

$$
\chi_{a}(X, \mathcal{F}):=\chi\left(H^{*}(X, \mathcal{F})\right)
$$

and similarly for $\chi_{a}\left(X^{(n)}, \mathcal{F}^{(n)}\right)$. Then

$$
\sum_{n \geq 0} \chi_{a}\left(X^{(n)}, \mathcal{F}^{(n)}\right) \cdot t^{n}=(1-t)^{-\chi_{a}(X, \mathcal{F})}=\exp \left(\sum_{r \geq 1} \chi_{a}(X, \mathcal{F}) \cdot \frac{t^{r}}{r}\right) .
$$

(c) Let $X$ and $\mathcal{F}$ be as in (a), with $X$ compact and $\mathcal{F}$ self-dual with respect to Verdier duality, so that $H^{0}(X, \mathcal{F})$ gets an induced symmetric (non-degenerate) pairing. Assume $\mathbb{K} \subset \mathbb{R}$ and let

$$
\sigma(X, \mathcal{F}):=\sigma\left(H^{0}(X, \mathcal{F})\right)
$$

be the corresponding signature, and similarly for $\sigma\left(X^{(n)}, \mathcal{F}^{(n)}\right)$. Then

$$
\sum_{n \geq 0} \sigma\left(X^{(n)}, \mathcal{F}^{(n)}\right) \cdot t^{n}=\frac{(1+t)^{\frac{\sigma(X, \mathcal{F})-\chi(X, \mathcal{F})}{2}}}{(1-t)^{\frac{\sigma(X, \mathcal{F})+\chi(X, \mathcal{F})}{2}}} .
$$

Note that our coefficients $\mathcal{M} \in D^{b} \operatorname{MHM}(X)$, e.g. $\mathbb{Q}_{X}^{H}$ or $I C_{X}^{\prime H}(\mathcal{L})$, are in general highly complicated objects. For this reason, we give a proof of our results based only on suitable abstract formal properties of the category $D^{b} \operatorname{MHM}(X)$, all of which are contained in the very deep work of M. Saito ([35, 36, 37]). And in this form it applies to other interesting situations as well (like Theorem 1.4). Moreover, the key underlying structures become much better visible in this abstract context. Of course the price we have to pay is exactly this abstraction. But in a forthcoming paper we will show, that exactly this formalism is needed for the characteristic class version of our results from this paper.

In Section 2, we explain the relation between such generating series for suitable invariants and pre-lambda structures on the coefficient theory on a point space. This will give a very quick proof of our results. Moreover, it suggests to also consider the corresponding alternating projector

$$
\mathcal{P}=\mathcal{P}^{a l t}=(-)^{\operatorname{sign}-\Sigma_{n}}:=\frac{1}{n !} \sum_{\sigma \in \Sigma_{n}}(-1)^{\operatorname{sign}(\sigma)} \cdot \psi_{\sigma}
$$

onto the alternating $\Sigma_{n}$-equivariant sub-object (with $\psi_{\sigma}$ given by the $\Sigma_{n}$-action as before).

For a mixed Hodge module complex $\mathcal{M} \in D^{b} \mathrm{MHM}(X)$ on the complex quasi-projective variety $X$ we get a corresponding alternating object

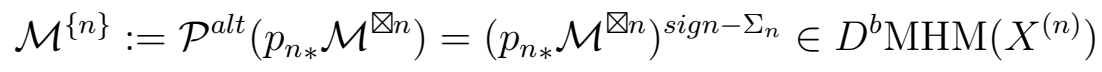


with the following additional property (cf. Lemma 5.7):

$$
j_{!} j^{*} \mathcal{M}^{\{n\}} \simeq \mathcal{M}^{\{n\}} \text { and } i^{*} \mathcal{M}^{\{n\}} \simeq 0
$$

were $j: B(X, n)=X^{\{n\}}:=F(X, n) / \Sigma_{n} \rightarrow X^{(n)}$ is the open inclusion of the configuration space $B(X, n)=X^{\{n\}}$ of all unordered $n$-tuples of different points in $X$. Finally, $i$ is the closed inclusion of the complement of $X^{\{n\}}$ into $X^{(n)}$. So in this case one has a Künneth formula

$$
H_{c}^{*}\left(X^{\{n\}}, \mathcal{M}^{\{n\}}\right) \simeq\left(H_{c}^{*}\left(X^{n}, \mathcal{M}^{\bigotimes n}\right)\right)^{s i g n-\Sigma_{n}} \simeq\left(\left(H_{c}^{*}(X, \mathcal{M})\right)^{\otimes n}\right)^{s i g n-\Sigma_{n}}
$$

for the cohomology with compact support. Again these are isomorphisms of graded groups of mixed Hodge structures and, for suitable choices, $\mathcal{M}^{\{n\}} \mid X^{\{n\}}$ can become a highly interesting object on the configuration space $B(X, n)=X^{\{n\}}$ of all unordered $n$-tuples of different points in $X$.

For example, $\left(\mathbb{Q}_{X}^{H}\right)^{\{n\}} \mid X^{\{n\}}$ is a mixed Hodge module complex, whose underlying rational sheaf complex is just the rank-one locally constant sheaf $\epsilon_{n}$ on $X^{\{n\}}$, corresponding to the sign-representation of $\pi_{1}\left(X^{\{n\}}\right)$ induced by the quotient homomorphism $\pi_{1}\left(X^{\{n\}}\right) \rightarrow \Sigma_{n}$ of the Galois covering $F(X, n) \rightarrow X^{\{n\}}$. So for $X$ smooth we can think of $\left(\mathbb{Q}_{X}^{H}\right)^{\{n\}} \mid X^{\{n\}}$ just as the corresponding variation of pure Hodge structures $\epsilon_{n}$ (of weight 0 ) on the smooth variety $X^{\{n\}}$. Similarly, one gets for a pure dimensional quasi-projective variety $X$, with $\mathcal{L}$ is an admissible (graded polarizable) variation of mixed Hodge structures with quasi-unipotent monodromy at infinity defined on a smooth Zariski open dense subset of $X$ (cf. Remark 5.6):

$$
\left(I C^{\prime H}(\mathcal{L})\right)^{\{n\}}=I C^{\prime H}{ }^{(n)}\left(\epsilon_{n} \otimes \mathcal{L}^{(n)}\right),
$$

with $\epsilon_{n}$ the corresponding local system on the open dense smooth subvariety $X_{r e g}^{\{n\}}$ of $X^{\{n\}}$. These examples provide interesting coefficients that can be used in the following result (for the special case of constant coefficients $\mathcal{M}=\mathbb{Q}_{X}^{H}$ compare with [15][Cor.5.7]).

Corollary 1.5. Let $X$ be a complex quasi-projective variety and $\mathcal{M} \in D^{b} M H M(X)$ a bounded complex of mixed Hodge modules on $X$. Then the following formulae hold for the generating series of invariants:

$$
\begin{aligned}
& \sum_{n \geq 0}\left(\sum_{p, q, k} h_{c}^{p, q, k}\left(X^{\{n\}}, \mathcal{M}^{\{n\}}\right) \cdot y^{p} x^{q}(-z)^{k}\right) \cdot t^{n}=\prod_{p, q, k}\left(1+y^{p} x^{q} z^{k} t\right)^{(-1)^{k} \cdot h_{c}^{p, q, k}(X, \mathcal{M})} \\
& \sum_{n \geq 0} e_{c}\left(X^{\{n\}}, \mathcal{M}^{\{n\}}\right) \cdot t^{n}=\prod_{p, q}\left(1+y^{p} x^{q} t\right)^{e_{c}^{p, q}(X, \mathcal{M})} \\
&=\exp \left(-\sum_{r \geq 1} e_{c}(X, \mathcal{M})\left(y^{r}, x^{r}\right) \cdot \frac{(-t)^{r}}{r}\right)
\end{aligned}
$$




$$
\begin{aligned}
\sum_{n \geq 0} \chi_{-y}^{c}\left(X^{\{n\}}, \mathcal{M}^{\{n\}}\right) \cdot t^{n} & =\prod_{p}\left(1+y^{p} t\right)^{f_{c}^{p}(X, \mathcal{M})} \\
& =\exp \left(-\sum_{r \geq 1} \chi_{-y^{r}}^{c}(X, \mathcal{M}) \cdot \frac{(-t)^{r}}{r}\right) .
\end{aligned}
$$

More generally, we follow in Section 2 Atiyah's approach [1] to power operations in $K$-theory. In this way we can extend operations like symmetric or alternating powers from operations on the "coefficient pre-lambda ring" to a method of introducing very interesting coefficients like $\mathcal{M}^{(n)}, \mathcal{M}^{\{n\}}$ on the symmetric products $X^{(n)}$. In particular we get such coefficients related to the corresponding Adams operations $\Psi_{r}$ ! In Section 3 we explain another proof of Corollary 1.2 based on equivariant genera and (abstract) "trace homomorphisms". This second proof will be generalized in a forthcoming paper to get the corresponding results for characteristic classes. Finally, two appendices are used to keep some technicalities out of the way. In appendix A we collect in an abstract form the categorical notions needed to formulate and prove our main results about the relation between exterior products and equivariant objects. We also indicate why this can be applied to our main examples of derived categories like $D^{b} \operatorname{MHM}(X), D_{c}^{b}(X)$ and $D_{c o h}^{b}(X)$. In appendix B we collect the technical points about (the derived category of) mixed Hodge modules needed for our results in this introduction.

Acknowledgements. The authors thank Sylvain Cappell and Shoji Yokura for discussions on the subject of this paper. The authors also acknowledge the support of New York University, where this work was started.

\section{Symmetric PROdUCTS AND PRE-LAMbDA RINGS}

2.1. Pre-lambda rings. We work on an underlying geometric category space of spaces (with finite products $\times$ and terminal object $p t$ ), e.g., the category of complex quasiprojective varieties (or compact topological or complex analytic spaces) with coefficients in a category $A(-)$ satisfying the following assumptions:

$(\mathbb{Q}$-lin) $A(-)$ is a pseudo-abelian $\mathbb{Q}$-linear additive category, together with

(ext) a suitable $\mathbb{Q}$-bilinear "exterior product" structure

$$
\bigotimes: A(X) \times A(Y) \rightarrow A(X \times Y)
$$

(psf) a covariant $\mathbb{Q}$-linear pseudofunctor $f_{*}: A(X) \rightarrow A(Y)$ for $f: X \rightarrow Y$ a morphism in space,

(Kue) A Künneth morphism

$$
\text { Kue }: f_{*} M \otimes f_{*}^{\prime} M^{\prime} \rightarrow\left(f \times f^{\prime}\right)_{*}\left(M \otimes M^{\prime}\right)
$$

for morphisms $f: X \rightarrow Y, f^{\prime}: X^{\prime} \rightarrow Y^{\prime}$ and $M \in A(X), M^{\prime} \in A\left(X^{\prime}\right)$.

And these have to satisfy suitable compatibilites as explained in Appendix A. In categorical terms it means that the cofibered category $A^{o p} /$ space associated to the covariant 
pseudofunctor $(-)_{*}$ (with objects $(X, \mathcal{M})$ for $X \in o b($ space) and $\mathcal{M} \in A(X))$ becomes a symmetric monoidal category with the product

$$
(X, M) \otimes\left(X^{\prime}, M^{\prime}\right):=\left(X \times X^{\prime}, M \otimes M^{\prime}\right)
$$

such that the projection functor onto the first component is a strict monoidal functor. Then the $n$-th exterior product

$$
\mathcal{M}^{\bigotimes n} \in A_{\Sigma_{n}}\left(X^{n}\right)
$$

becomes a well-defined $\Sigma_{n}$-equivariant object on $X^{n}$.

As explained in the appendices $\mathrm{A}$ and $\mathrm{B}$, this is for example the case in the quasiprojective context for $A(X)=D^{b} \operatorname{MHM}(X)$, with $(-)_{*}$ the push down (with proper support) $f_{*}$ (or $f_{!}$). Similarly for $A(X)=D_{c}^{b}(X)$ or $D_{c o h}^{b}(X)$ in the context of Theorem 1.4, again with $(-)_{*}$ the derived push down (with proper support) $f_{*}:=R f_{*}$ (or $\left.f_{!}:=R f_{!}\right)$.

To get an important Künneth formula like (9) above, we assume that the Künneth morphism is an isomorphism, at least for constant morphisms $k: X \rightarrow p t$ to the terminal object $p t \in o b($ space). Again this is allways the case in our examples (compare with the references given in appendix $\mathrm{A}$ ). This allows the calculation of our invariants for $\mathcal{M} \in A(X)$ in terms of those for $k_{*} \mathcal{M} \in A(p t)$, since then

$$
\left(k^{n}\right)_{*}\left(\mathcal{M}^{\bigotimes n}\right) \simeq\left(k_{*} \mathcal{M}\right)^{\bigotimes n} \in A_{\Sigma_{n}}(p t) .
$$

Finally, the "pseudo-abelian $\mathbb{Q}$-linear additive" structure is used for defining the projector

$$
\mathcal{P}=\mathcal{P}^{s y m}=\frac{1}{n !} \sum_{\sigma \in \Sigma_{n}} \psi_{\sigma}=(-)^{\Sigma_{n}}
$$

onto the $\Sigma_{n}$-invariant sub-object

$$
\mathcal{M}^{(n)}:=\mathcal{P}\left(p_{n_{*}} \mathcal{M}^{\otimes n}\right) \in A\left(X^{(n)}\right)
$$

of the equivariant object

$$
p_{n *} \mathcal{M}^{\bigotimes n} \in A_{\Sigma_{n}}\left(X^{(n)}\right),
$$

with $p_{n}$ the projection on the symmetric product $X^{(n)}$, and the isomorphisms $\psi_{\sigma}$ coming from the symmetric group action (as explained in appendix A). We call $\mathcal{M}^{(n)}$ the $n$-th symmetric product of $\mathcal{M}$. Note that the image of an object acted upon by a projector $\mathcal{P}$ as above is functorial under $\Sigma_{n}$-equivariant morphisms. For example, if we let

$$
k: X^{(n)} \rightarrow \mathrm{pt}
$$

be the constant map to a point, then for any $\mathcal{M} \in A(X)$ we have:

$$
k_{*}\left(\mathcal{M}^{(n)}\right)=k_{*}\left(\mathcal{P}\left(p_{n_{*}} \mathcal{M}^{\bigotimes n}\right)\right)=\mathcal{P}\left(k_{*} p_{n *} \mathcal{M}^{\bigotimes n}\right) \simeq \mathcal{P}\left(\left(k \circ p_{n}\right)_{*} \mathcal{M}^{\bigotimes n}\right) \in A(p t) .
$$

Note that we do not need an "interior product", i.e. a "tensor structure" on $A(-)$ for a space $X^{n}$ or $X^{(n)}$, which in general doesn't exist (e.g. for perverse sheaves, coherent 
sheaves or a compatible duality on singular spaces). But on a point space $p t$, we get from the "exterior product" such a "tensor structure" $\otimes$ so that on a point we are working with a "pseudo-abelian $\mathbb{Q}$-linear tensor category" $(A(p t), \otimes)$. And this is important, since it gives a canonical pre-lambda structure on $\bar{K}_{0}(A(p t))([23])$, which will be the key for our results :

$$
\sigma_{t}: \bar{K}_{0}(A(p t)) \rightarrow \bar{K}_{0}(A(p t))[[t]] ; \quad[\mathcal{V}] \mapsto 1+\sum_{n \geq 1}\left[\left(\mathcal{V}^{\otimes n}\right)^{\Sigma_{n}}\right] \cdot t^{n}
$$

In the applications we will take $\mathcal{V}=k_{*} \mathcal{M}$, for which (by using (32) and (34)) we have:

$$
\left(\mathcal{V}^{\otimes n}\right)^{\Sigma_{n}} \simeq k_{*}\left(\mathcal{M}^{(n)}\right) \text {. }
$$

Here $\bar{K}_{0}(-)$ denotes the Grothendieck group of an additive category viewed as an exact category by the split exact sequences corresponding to direct sums $\oplus$, i.e. the Grothendieck group associated to the abelian monoid of isomorphism classes of objects with the direct sum. Then $\bar{K}_{0}(A(p t))$ becomes a commutative ring with unit $1_{p t}$ and product induced by $\otimes$, whereas $\bar{K}_{0}(A(X))$ becomes a $\bar{K}_{0}(A(p t))$-module by $\otimes$. And a pre-lambda structure on a commutative ring $R$ with unit 1 just means a group homomorphism

$$
\sigma_{t}:(R,+) \rightarrow(R[[t]], \cdot) ; r \mapsto 1+\sum_{n \geq 1} \sigma_{n}(r) \cdot t^{n}
$$

with $\sigma_{1}=i d_{R}$, where "." on the target side denotes the multiplication of formal power series. So this corresponds to a family of selfmaps $\sigma_{n}: R \rightarrow R\left(n \in \mathbb{N}_{0}\right)$ satisfying for all $r \in R$ :

$$
\sigma_{0}(r)=1, \sigma_{1}(r)=r \quad \text { and } \quad \sigma_{k}(r)=\sum_{i+j=k} \sigma_{i}(r) \cdot \sigma_{j}(r)
$$

Remark 2.1. In the case when $A(p t)$ is also an abelian category with $\otimes$ exact in both variables, this pre-lambda structure even descends to the usual Grothendieck group

$$
K_{0}(A(p t))=\bar{K}_{0}(A(p t)) /(e x-s e q)
$$

with direct sum for all short exact sequences. For a short exact sequence

$$
0 \rightarrow \mathcal{V}^{\prime} \rightarrow \mathcal{V} \rightarrow \mathcal{V}^{\prime \prime} \rightarrow 0 \quad \text { in } A(p t)
$$

one can introduce an increasing two step filtration on $\mathcal{V}$ :

$$
F: F^{0} \mathcal{V}:=\mathcal{V}^{\prime} \subset F^{1} \mathcal{V}=: \mathcal{V}, \text { with } G r_{0}^{F} \mathcal{V} \simeq \mathcal{V}^{\prime}, G r_{1}^{F} \mathcal{V} \simeq \mathcal{V}^{\prime \prime}
$$

Then by the exactness of $\otimes$ and of the projector $\mathcal{P}$ one gets an induced filtration $F$ on $\left(\mathcal{V}^{\otimes n}\right)^{\Sigma_{n}}$ with (compare [13] [Part II, sec.1.1]):

$$
G r_{*}^{F}\left(\left(\mathcal{V}^{\otimes n}\right)^{\Sigma_{n}}\right) \simeq\left(\left(G r_{*}^{F} \mathcal{V}\right)^{\otimes n}\right)^{\Sigma_{n}}
$$

so that

$$
\left[\left(\mathcal{V}^{\otimes n}\right)^{\Sigma_{n}}\right]=\left[G r_{*}^{F}\left(\left(\mathcal{V}^{\otimes n}\right)^{\Sigma_{n}}\right)\right]=\left[\left(\left(G r_{*}^{F} \mathcal{V}\right)^{\otimes n}\right)^{\Sigma_{n}}\right] \in K_{0}(A(p t))
$$


Similarly, for the (symmetric bimonoidal) category space one gets on the Grothendieck group $\left(\bar{K}_{0}(\right.$ space $\left.), \cup\right)$ associated to the abelian monoid of isomorphism classes of objects with the sum coming from the disjoint union $\cup$ (or categorical coproduct) and the product induced by $\times$, the structure of a commutative ring with unit $[p t]$ and zero $[\emptyset]$. And for our category space $=\operatorname{var} / \mathbb{C}$ of complex quasi-projective varieties (or compact topological or complex analytic spaces) we also get a pre-lambda ring structure on $\bar{K}_{0}(\operatorname{var} / \mathbb{C})$ by the Kapranov zeta function $([24])$ :

$$
\sigma_{t}: \bar{K}_{0}(\operatorname{var} / \mathbb{C}) \rightarrow \bar{K}_{0}(\operatorname{var} / \mathbb{C})[[t]] ;[X] \mapsto 1+\sum_{n \geq 1}\left[X^{(n)}\right] \cdot t^{n},
$$

because $(X \cup Y)^{(n)} \simeq \bigcup_{i+j=n}\left(X^{(i)} \times Y^{(j)}\right)$. In fact this pre-lambda ring structure factorizes in the context of complex quasi-projective varieties also over the motivic Grothendieck group

$$
K_{0}(\operatorname{var} / \mathbb{C}):=\bar{K}_{0}(\operatorname{var} / \mathbb{C}) /(a d d)
$$

defined as the quotient by the additivity relation $[X]=[Z]+[X \backslash Z]$ for $Z \subset X$ a closed complex subvariety.

Finally, also the Laurent polynomial $\operatorname{ring} \mathbb{Z}\left[x_{1}^{ \pm 1}, \ldots, x_{r}^{ \pm 1}\right]$ in $r$ variables $(r \geq 0)$ becomes a pre-lambda ring (compare e.g. [19] and [22][p.578]) by

$$
\sigma_{t}\left(\sum_{\vec{k} \in \mathbb{Z}^{n}} a_{\vec{k}} \cdot \vec{x} \vec{k}\right):=\prod_{\vec{k} \in \mathbb{Z}^{n}}\left(1-\vec{x}^{\vec{k}} \cdot t\right)^{-a_{\vec{k}}}
$$

Since the cohomolgy (with compact support) is additive under disjoint union:

$$
H_{(c)}^{*}(X \cup Y, \mathbb{Q})=H_{(c)}^{*}(X, \mathbb{Q}) \oplus H_{(c)}^{*}(Y, \mathbb{Q}),
$$

we get in the context of complex quasi-projective varieties a group homomorphism

$$
h_{(c)}: \bar{K}_{0}(\operatorname{var} / \mathbb{C}) \rightarrow \mathbb{Z}\left[y^{ \pm 1}, x^{ \pm 1}, z^{ \pm 1}\right] ;[X] \mapsto \sum_{p, q, k} h_{(c)}^{p, q, k}(X) \cdot y^{p} x^{q}(-z)^{k},
$$

with $h_{(c)}^{p, q, k}(X):=h^{p, q}\left(H_{(c)}^{k}(X, \mathbb{Q})\right)$. And by the usual Künneth isomorphisms (which respects the underlying mixed Hodge structures of Deligne), $h_{(c)}$ is even a ring homomorphism! Then the generating series (5) for $h_{c}$ (or its counterpart for $h$ following from Theorem 1.1) just tells us the well known fact (compare [19]) that $h_{(c)}$ is a morphism of pre-lambda rings! And the corresponding morphism of pre-lambda rings $e_{c}$ factorizes over the motivic Grothendieck ring:

$$
e_{c}: K_{0}(\operatorname{var} / \mathbb{C}) \rightarrow \mathbb{Z}\left[y^{ \pm 1}, x^{ \pm 1}\right] ;[X] \mapsto \sum_{p, q} e_{c}^{p, q}(X) \cdot y^{p} x^{q}
$$

because the long exact sequence for the cohomology with compact support

$$
\cdots \rightarrow H_{c}^{k}(X \backslash Z, \mathbb{Q}) \rightarrow H_{c}^{k}(X, \mathbb{Q}) \rightarrow H_{c}^{k}(Z, \mathbb{Q}) \rightarrow \cdots
$$


for $Z \subset X$ a closed subvariety is an exact sequence of mixed Hodge structures. (For applications of this to the associated power structures compare with [19].)

Theorem 1.1 asserts that we can factorize these as homomorphisms of pre-lambda rings

$$
h: \bar{K}_{0}(\operatorname{var} / \mathbb{C}) \stackrel{k_{*}\left(\mathbb{Q}_{?}^{H}\right)}{\longrightarrow} \bar{K}_{0}\left(D^{b} \operatorname{MHM}(p t)\right) \stackrel{h}{\longrightarrow} \mathbb{Z}\left[y^{ \pm 1}, x^{ \pm 1}, z^{ \pm 1}\right]
$$

and

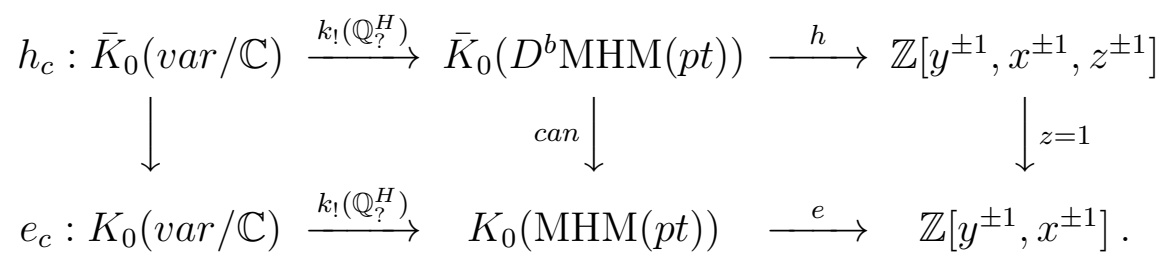

Here $K_{0}(\mathrm{MHM}(p t))$ is the Grothendieck group of the abelian category $\operatorname{MHM}(p t)$, with can induced by the alternating sum of cohomology objects of a complex. The fact that the group homomorphisms on the left side (compare [10] [sec.5]):

$$
[X] \mapsto\left[k_{?}\left(\mathbb{Q}_{X}^{H}\right)\right] \quad \text { for } ?=*, !
$$

are ring homomorphisms of pre-lambda rings follows from (14), (32) and (34).

Note that the homomorphism $h: \bar{K}_{0}\left(D^{b} \operatorname{MHM}(p t)\right) \rightarrow \mathbb{Z}\left[y^{ \pm 1}, x^{ \pm 1}, z^{ \pm 1}\right]$ can further be factorized as

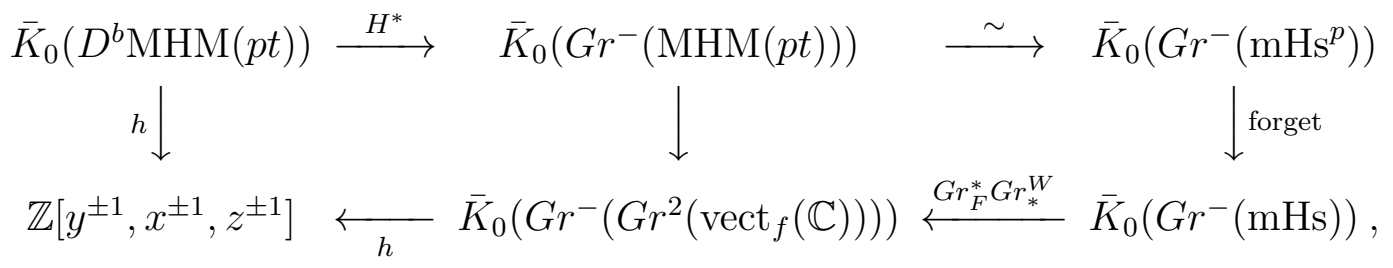

where the following notations are used:

(a) For an additive (or abelian) tensor category $(A, \otimes), G r^{-}(A)$ denotes the additive (or abelian) tensor category of bounded graded objects in $A$, i.e. functors $G: \mathbb{Z} \rightarrow$ $A$, with $G_{n}:=G(n)=0$ except for finitely many $n \in \mathbb{Z}$. Here

$$
\left(G \otimes G^{\prime}\right)_{n}:=\oplus_{i+j=n} G_{i} \otimes G_{j},
$$

with the Koszul symmetry isomorphism (indicated by the - sign in $G r^{-}$):

$$
(-1)^{i \cdot j} s\left(G_{i}, G_{j}\right): G_{i} \otimes G_{j} \simeq G_{j} \otimes G_{i} .
$$

(b) $H^{*}: D^{b} \operatorname{MHM}(p t) \rightarrow G r^{-}(\operatorname{MHM}(p t))$ is the total cohomology functor $\mathcal{V} \mapsto$ $\oplus_{n} H^{n}(\mathcal{V})$. Note that this is a functor of additive tensor categories (i.e. commutes with direct sums $\oplus$ and tensor products $\otimes$ ), if we choose the Koszul symmetry isomorphism on $\mathrm{Gr}^{-}(\mathrm{MHM}(p t))$. In fact $D^{b} \mathrm{MHM}(p t)$ is a triangulated category with bounded $t$-structure satisfying [6][Def.4.2] so that the claim follows from [6] [thm.4.1, cor.4.4]. 
(c) The isomorphism $\mathrm{MHM}(p t) \simeq \mathrm{mHs}^{p}$ from Saito's work [35, 36] was already mentioned in the introduction.

(d) forget : $\mathrm{mHs}^{p} \rightarrow \mathrm{mHs}$ is the functor of forgetting that the corresponding $\mathbb{Q}$-mixed Hodge structure is graded polarizable.

(e) $G r_{F}^{*} G r_{*}^{W}: \mathrm{mHs} \rightarrow G r^{2}\left(\operatorname{vect}_{f}(\mathbb{C})\right)$ is the functor of taking the associated bigraded finite dimensional $\mathbb{C}$-vector space

$$
\text { mHs } \ni \mathcal{V} \mapsto \oplus_{p, q} G r_{F}^{p} G r_{p+q}^{W}\left(\mathcal{V} \otimes_{\mathbb{Q}} \mathbb{C}\right) \in G r^{2}\left(\operatorname{vect}_{f}(\mathbb{C})\right)
$$

This is again a functor of additive tensor categories, if this time we use the induced symmetry isomorphism without any sign changes.

(f) $h: G r^{-}\left(G r^{2}\left(\operatorname{vect}_{f}(\mathbb{C})\right)\right) \rightarrow \mathbb{Z}\left[y^{ \pm 1}, x^{ \pm 1}, z^{ \pm 1}\right]$ is given by taking the dimension counting Laurent polynomial

$$
\oplus\left(V^{p, q}\right)^{k} \mapsto \sum_{p, q, k} \operatorname{dim}\left(\left(V^{p, q}\right)^{k}\right) \cdot y^{p} x^{q}(-z)^{k},
$$

with $k$ the degree with respect to the grading in $G r^{-}$. In fact this corresponds to the sign choice of numbering by $(-z)^{k}$ in this definition.

Remark 2.2. The fact that total cohomology functor $H^{*}: D^{b} M H M(p t) \rightarrow G r^{-}(M H M(p t))$ is a tensor functor corresponds to the Künneth formula

$$
H^{*}\left(\mathcal{V}^{\otimes n}\right) \simeq\left(H^{*}(\mathcal{V})\right)^{\otimes n} \quad \text { for } \mathcal{V} \in D^{b} M H M(p t) .
$$

Together with (32) and (34) this implies the important Künneth isomorphism (9) from the introduction.

Note that all functors in (a)-(e) are functors of $\mathbb{Q}$-linear tensor categories, so that they induce ring homomorphisms of the corresponding Grothendieck groups $\bar{K}_{0}(-)$ respecting the corresponding pre-lambda structures (35). Therefore, Theorem 1.1 follows from the following

Proposition 2.3. The ring homomorphism

$$
h: \bar{K}_{0}\left(G r^{-}\left(G r^{2}\left(\operatorname{vect}_{f}(\mathbb{C})\right)\right)\right) \rightarrow \mathbb{Z}\left[y^{ \pm 1}, x^{ \pm 1}, z^{ \pm 1}\right]
$$

is a homomorphism of pre-lambda rings.

Proof. Since $h$ is a ring homomorphism, it is enough to check the formula

$$
\sigma_{t}(h(L))=1+\sum_{n \geq 1} h\left(\left(L^{\otimes n}\right)^{\Sigma_{n}}\right) \cdot t^{n}
$$

for the set of generators given by the one dimensional graded vector spaces $L=\left(L^{p, q}\right)^{k}=$ : $L^{p, q, k}$ sitting in degree $(p, q, k)$. Here we have to consider the two cases when $k$ is even or odd, each giving a different meaning to $\left((-)^{\otimes n}\right)^{\Sigma_{n}}$ because of the graded commutativity. For $k$ even, $\left((-)^{\otimes n}\right)^{\Sigma_{n}}$ corresponds to the $n$-th symmetric power $\operatorname{Sym}_{n}(-)$, whereas for $k$ odd it corresponds to the $n$-th alternating power $A l t_{n}(-)$. For $k$ even we get

$$
\left(L^{\otimes n}\right)^{\Sigma_{n}}=\operatorname{Sym}_{n}(L)=L^{\otimes n}, \quad \text { with } \quad h\left(L^{\otimes n}\right)=\left(y^{p} x^{q} z^{k}\right)^{n},
$$


thus

$$
1+\sum_{n \geq 1} h\left(\left(L^{\otimes n}\right)^{\Sigma_{n}}\right) \cdot t^{n}=1+\sum_{n \geq 1}\left(y^{p} x^{q} z^{k} t\right)^{n}=\left(1-y^{p} x^{q} z^{k} t\right)^{-1} .
$$

For $k$ odd we get

$$
\left(L^{\otimes n}\right)^{\Sigma_{n}}=A l t_{n}(L)= \begin{cases}0 & \text { for } n>1 \\ L & \text { for } n=1\end{cases}
$$

Therefore

$$
1+\sum_{n \geq 1} h\left(\left(L^{\otimes n}\right)^{\Sigma_{n}}\right) \cdot t^{n}=1-y^{p} x^{q} z^{k} t .
$$

Then Proposition 2.3 follows, since by the definition (37) we get

$$
\sigma_{t}\left(1+y^{p} x^{q} z^{k}\right)=\left(1-y^{p} x^{q} z^{k} t\right)^{-1} \quad \text { and } \quad \sigma_{t}\left(1-y^{p} x^{q} z^{k}\right)=1-y^{p} x^{q} z^{k} t .
$$

Exactly the same method also gives a proof of Theorem 1.4(a) and (b), if one uses (32), (34) for $A(X)=D_{c}^{b}(X)$ and $A(X)=D_{c o h}^{b}(X)$, respectively, together with the factorizations

$$
\bar{K}_{0}\left(D_{c}^{b}(p t)\right) \stackrel{H^{*}}{\longrightarrow} \bar{K}_{0}\left(G r^{-}\left(\operatorname{vect}_{f}(\mathbb{C})\right)\right) \stackrel{\chi}{\longrightarrow} \mathbb{Z}
$$

and resp.

$$
\bar{K}_{0}\left(D_{\text {coh }}^{b}(p t)\right) \stackrel{H^{*}}{\longrightarrow} \bar{K}_{0}\left(G r^{-}\left(\operatorname{vect}_{f}(\mathbb{C})\right)\right) \stackrel{\chi}{\longrightarrow} \mathbb{Z} .
$$

Here $\chi$ is the usual Euler charcateristic, with $H^{*}$ once more a functor of tensor categories by [6] [thm.41, cor.4.4]. And $\mathbb{Z}$ is endowed with the pre-lambda structure $\sigma_{t}(a)=(1-t)^{-a}$. Only the proof of Theorem 1.4(c) needs a small twist. We use the factorization

$$
\bar{W}_{0}\left(D_{c}^{b}(p t)\right) \stackrel{H^{*}}{\longrightarrow} \bar{W}_{0}\left(G r^{-}\left(\operatorname{vect}_{f}(\mathbb{K})\right)\right) \stackrel{\sigma}{\longrightarrow} \mathbb{Z} .
$$

Here $\bar{W}_{0}(-)$ denotes the Grothendieck-Witt group of isometry classes of symmetric selfdual objects in the underlying additive category with duality, with addition coming once more from the direct sum $\oplus$. In other words this is the Grothendieck group associated to the abelian monoid of isomorphism classes of symmetric self-dual objects with the direct sum. On $D_{c}^{b}(X)$ we use Verdier duality with its natural biduality isomorphism. Note that Verdier duality commutes with the pushdown $f_{*}:=R f_{*}=R f_{\text {! }}$ for proper maps (e.g. a constant map on a compact space) and with exterior products (compare [10, 39]). So one gets induced transformations $f_{*}$, $\otimes$ on the level of Grothendieck-Witt groups. In particular $\bar{W}_{0}\left(D_{c}^{b}(p t)\right)$ becomes a commutative ring with unit [KK] and multiplication coming from $\otimes\left(\right.$ and $\bar{W}_{0}\left(D_{c}^{b}(X)\right)$ becomes a $\bar{W}_{0}\left(D_{c}^{b}(p t)\right)$-module). On $G r^{-}\left(\operatorname{vect}_{f}(\mathbb{K})\right)$ we use the naive duality $(-)^{\vee}$ of finite dimensional vector spaces (with the corresponding biduality isomorphism), so that the transformation $H^{*}$ commutes with duality. Finally the ring homomorphism

$$
\sigma: \bar{W}_{0}\left(\left(\operatorname{vect}_{f}(\mathbb{K})\right) \rightarrow \mathbb{Z}\right.
$$


is given by the signature of the underlying vector space with symmetric duality pairing. Here we are working with vector spaces over a base field $\mathbb{K} \subset \mathbb{R}$. Note that $(-)^{\vee}$ : $H^{i}(\mathcal{V}) \rightarrow H^{-i}(\mathcal{V})$ so that $\oplus_{i \neq 0} H^{i}(\mathcal{V})$ is a hyperbolic direct summand of $H^{*}(\mathcal{V})$ and

$$
\sigma\left(H^{*}(\mathcal{V})\right)=\sigma\left(H^{0}(\mathcal{V})\right)
$$

The Grothendieck-Witt rings $\bar{W}_{0}\left(D_{c}^{b}(p t)\right)$ and $\bar{W}_{0}\left(G r^{-}\left(\operatorname{vect}_{f}(\mathbb{K})\right)\right)$ get the structure of pre-lambda rings using the same definition of $\sigma_{t}$ via symmetric powers (e.g. compare [31, 32])

$$
\operatorname{Sym}_{n}(-)=\left((-)^{\otimes n}\right)^{\Sigma_{n}}
$$

(Note that this doesn't factorize over the usual Witt ring $W_{0}(-)=\bar{W}_{0}(-) /($ hyperbolic) which one gets by dividing out the isometry classes of hyperbolic spaces.) Moreover, the map induced by $H^{*}$ is compatible with these pre-lambda ring structures, since $H^{*}$ also commutes with duality and $\otimes$. Then Theorem 1.4(c) follows from the following

Proposition 2.4. The following formula holds for $[\mathcal{V}] \in \bar{W}_{0}\left(G r^{-}\left(\operatorname{vect}_{f}(\mathbb{K})\right)\right)$ :

$$
1+\sum_{n \geq 1} \sigma\left(\left(\mathcal{V}^{\otimes n}\right)^{\Sigma_{n}}\right) \cdot t^{n}=\frac{(1+t)^{\frac{\sigma(\mathcal{V})-\chi(\mathcal{V})}{2}}}{(1-t)^{\frac{\sigma(\mathcal{V})+\chi(\mathcal{V})}{2}}} \in \mathbb{Z}[[t]]
$$

with $\chi: \bar{W}_{0}\left(G r^{-}\left(\right.\right.$vect $\left.\left._{f}(\mathbb{K})\right)\right) \rightarrow \mathbb{Z}$ the Euler characteristic of the underlying graded vector space.

Proof. Since $\sigma, \chi$ are ring homomorphisms, it is enough to check that formula (40) holds for a set of generators of $\bar{W}_{0}\left(G r^{-}\left(\operatorname{vect}_{f}(\mathbb{K})\right)\right)$. Indeed, both sides of (40) behave correctly under direct sums. Here we work with the following set of generators:

(a) A one dimensional graded vector spaces $\mathcal{V}=L$ sitting in degree $k=0$, with $\sigma(L)= \pm 1$ and $\chi(L)=1$.

(b) A two dimensional hyperbolic graded vector space $\mathcal{V}=L \oplus L^{\vee}$, with $L$ sitting in degree $k>0$ so that $\sigma(\mathcal{V})=0$ and $\chi(\mathcal{V})=(-1)^{k} \cdot 2$.

Again we have to consider the two cases when $k$ is even or odd, each giving different meaning to $\left((-)^{\otimes n}\right)^{\Sigma_{n}}$ because of the graded commutativity. For $k$ even, it corresponds to the $n$-th symmetric power $\operatorname{Sym}_{n}(-)$, whereas for $k$ odd it corresponds to the $n$-th alternating power $\operatorname{Alt}_{n}(-)$. In case (a) we get $\operatorname{Sym}_{n}(L)=L^{\otimes n}$ with $\sigma\left(L^{\otimes n}\right)=\sigma(L)^{n}$, so that

$$
1+\sum_{n \geq 1} \sigma\left(\left(L^{\otimes n}\right)^{\Sigma_{n}}\right) \cdot t^{n}=1+\sum_{n \geq 1}(\sigma(L) t)^{n} .
$$

For $\sigma(L)=1$ we get (with $\chi(L)=1)$ :

$$
1+\sum_{n \geq 1}(\sigma(L) t)^{n}=(1-t)^{-1}=\frac{(1+t)^{\frac{\sigma(L)-\chi(L)}{2}}}{(1-t)^{\frac{\sigma(L)+\chi(L)}{2}}},
$$


and for $\sigma(L)=-1$ we get (with $\chi(L)=1)$ :

$$
1+\sum_{n \geq 1}(\sigma(L) t)^{n}=(1+t)^{-1}=\frac{(1+t)^{\frac{\sigma(L)-\chi(L)}{2}}}{(1-t)^{\frac{\sigma(L)+\chi(L)}{2}}} .
$$

In case (b) we get for $k$ even (compare [32] [cor.5.2, prop.5.6]):

$$
\sigma\left(\operatorname{Sym}_{n}(\mathcal{V})\right)= \begin{cases}0 & , \text { for } n \text { odd } \\ 1 & , \text { for } n \text { even }\end{cases}
$$

Together with $\sigma(\mathcal{V})=0$ and $\chi(\mathcal{V})=2$ one gets

$$
1+\sum_{n \geq 1} \sigma\left(\left(\mathcal{V}^{\otimes n}\right)^{\Sigma_{n}}\right) \cdot t^{n}=\left(1-t^{2}\right)^{-1}=\frac{(1+t)^{\frac{\sigma(\mathcal{V})-\chi(\mathcal{V})}{2}}}{(1-t)^{\frac{\sigma(\mathcal{V})+\chi(\mathcal{V})}{2}}} .
$$

In case (b) we finally get for $k$ odd (compare [31] [cor.11.5, prop.11.6]):

$$
\sigma\left(\text { Alt }_{n}(\mathcal{V})\right)= \begin{cases}0 & , \text { for } n \text { odd or } n>2 \text { even } \\ -1 & , \text { for } n=2 .\end{cases}
$$

Together with $\sigma(\mathcal{V})=0$ and $\chi(\mathcal{V})=-2$ one gets

$$
1+\sum_{n \geq 1} \sigma\left(\left(\mathcal{V}^{\otimes n}\right)^{\Sigma_{n}}\right) \cdot t^{n}=1-t^{2}=\frac{(1+t)^{\frac{\sigma(\mathcal{V})-\chi(\mathcal{V})}{2}}}{(1-t)^{\frac{\sigma(\mathcal{V})+\chi(\mathcal{V})}{2}}} .
$$

Lets come back to abstract context. As soon as one has the pre-lambda structure $\sigma_{t}$ from (35) on $\bar{K}_{0}(A(p t))$, it is also natural to look at the opposite pre-lambda structure $\lambda_{t}:=\sigma_{-t}^{-1}($ cf. 23$]$ ):

$$
\lambda_{t}: \bar{K}_{0}(A(p t)) \rightarrow \bar{K}_{0}(A(p t))[[t]] ;[\mathcal{V}] \mapsto 1+\sum_{n \geq 1}\left[\left(\mathcal{V}^{\otimes n}\right)^{s i g n-\Sigma_{n}}\right] \cdot t^{n}
$$

with

$$
\mathcal{P}=\mathcal{P}^{\text {alt }}=(-)^{\operatorname{sign}-\Sigma_{n}}:=\frac{1}{n !} \sum_{\sigma \in \Sigma_{n}}(-1)^{\operatorname{sign}(\sigma)} \cdot \psi_{\sigma}
$$

the projector onto the alternating $\Sigma_{n}$-equivariant sub-object (and $\psi_{\sigma}$ as before). This opposite pre-lambda structure $\lambda_{t}$ is sometimes more natural, e.g. it is a lambda structure [23] [lem.4.1]. We call

$$
\mathcal{M}^{\{n\}}:=\mathcal{P}^{\text {alt }}\left(p_{n_{*}} \mathcal{M}^{\bigotimes n}\right) \in A\left(X^{(n)}\right)
$$

the $n$-th antisymmetric product of $\mathcal{M} \in A(X)$. For the constant map $k: X^{(n)} \rightarrow p t$ we get as in (34) (with $\left.\mathcal{P}=\mathcal{P}^{\text {alt }}\right)$ :

$$
k_{*}\left(\mathcal{M}^{\{n\}}\right)=k_{*}\left(\mathcal{P}\left(p_{n_{*}} \mathcal{M}^{\bigotimes n}\right)\right)=\mathcal{P}\left(k_{*} p_{n_{*}} \mathcal{M}^{\bigotimes n}\right) \simeq \mathcal{P}\left(\left(k \circ p_{n}\right)_{*} \mathcal{M}^{\bigotimes n}\right) \in A(p t),
$$

so that

$$
\left(\mathcal{V}^{\otimes n}\right)^{\operatorname{sign-\Sigma _{n}}} \simeq k_{*}\left(\mathcal{M}^{\{n\}}\right) \quad \text { for } \quad \mathcal{V}=k_{*} \mathcal{M} \in A(p t)
$$


Then Corollary 1.5 follows directly from (26), Theorem 1.1 and the relation $\lambda_{t}=\sigma_{-t}^{-1}$.

2.2. Adams operations for symmetric products. A natural way to extend such operations like symmetric or alternating powers from operations on the pre-lambda ring $\bar{K}_{0}(A(p t))$ (or $K_{0}(A(p t))$ ) to a method of introducing very important coefficients like $\mathcal{M}^{(n)}, \mathcal{M}^{\{n\}}$ on the symmetric products $X^{(n)}$, follows Atiyah's approach [1] to power operations in $K$-theory. In particular, one gets coefficients related to the corresponding Adams operations $\Psi_{r}$, which are behind the formulae (11),(13) (or their counterparts (30), (31) ), if these are formulated in terms of an exponential. For a pre-lambda ring $R$ this is the well-known formula (cf. [25, 15]):

$$
\sigma_{t}(a)=\sum_{n \geq 0} \sigma_{n}(a) \cdot t^{n}=\exp \left(\sum_{r \geq 1} \Psi_{r}(a) \cdot \frac{t^{r}}{r}\right) \in R \otimes_{\mathbb{Z}} \mathbb{Q}[[t]],
$$

following from the definition of $\Psi_{r}$ :

$$
\lambda_{t}(a)^{-1} \cdot \frac{d}{d t}\left(\lambda_{t}(a)\right)=: \frac{d}{d t}\left(\log \left(\lambda_{t}(a)\right)=: \sum_{r \geq 1}(-1)^{r-1} \Psi_{r}(a) \cdot t^{r-1} \in R[[t]]\right.
$$

by

$$
\sigma_{-t}(a)^{-1}=\lambda_{t}(a)=\exp \left(\sum_{r \geq 1}(-1)^{r-1} \Psi_{r}(a) \cdot \frac{t^{r}}{r}\right) \in R \otimes_{\mathbb{Z}} \mathbb{Q}[[t]] .
$$

Example 2.5. The Adams operation $\Psi_{r}$ on the Laurent polynomial ring $\mathbb{Z}\left[x_{1}^{ \pm 1}, \ldots, x_{k}^{ \pm 1}\right]$ in $k$ variables with the pre-lambda structure (37) is given by (compare e.g. [19]):

$$
\Psi_{r}\left(p\left(x_{1}, \cdots, x_{k}\right)\right)=p\left(x_{1}^{r}, \cdots, x_{k}^{r}\right),
$$

so that

$$
\begin{aligned}
\Psi_{r}\left(e_{(c)}(X, \mathcal{M})(y, x)\right) & =e_{(c)}(X, \mathcal{M})\left(y^{r}, x^{r}\right) \\
\text { and } \quad \Psi_{r}\left(\chi_{-y}^{(c)}(X, \mathcal{M})\right) & =\chi_{-y^{r}}^{(c)}(X, \mathcal{M}) .
\end{aligned}
$$

Assume now that $A(-)$ takes values in a Karoubian $\mathbb{Q}$-linear category. Since $\Sigma_{n}$ acts trivially on the symmetric product $X^{(n)}$, one has the following decomposition (compare [15] [thm.3.2])

$$
\bar{K}_{0}\left(A_{\Sigma_{n}}\left(X^{(n)}\right)\right) \simeq \bar{K}_{0}\left(A\left(X^{(n)}\right)\right) \otimes_{\mathbb{Z}} \operatorname{Rep}_{\mathbb{Q}}\left(\Sigma_{n}\right)
$$

for the Grothendieck group of $\Sigma_{n}$-equivariant objects in $A\left(X^{(n)}\right)$. In fact this follows directly from the corresponding decomposition of $\mathcal{V} \in A_{\Sigma_{n}}\left(X^{(n)}\right)$ by Schur functors $S_{\lambda}: A_{\Sigma_{n}}\left(X^{(n)}\right) \rightarrow A\left(X^{(n)}\right)([14,[23]):$

$$
\mathcal{V} \simeq \sum_{\lambda \vdash n} V_{\lambda} \otimes S_{\lambda}(\mathcal{V})
$$

with $V_{\lambda}$ the irreducible $\mathbb{Q}$-representation of $\Sigma_{n}$ corresponding to the partition $\lambda$ of $n$. 
This fact is used in the following definition of an operation

$$
\phi_{X}: o b(A(X)) \rightarrow \bar{K}_{0}\left(A\left(X^{(n)}\right)\right)
$$

associated to a group homomorphism

$$
\phi \in \operatorname{Rep}_{\mathbb{Q}}\left(\Sigma_{n}\right)_{*}:=\operatorname{hom}_{\mathbb{Z}}\left(\operatorname{Rep}_{\mathbb{Q}}\left(\Sigma_{n}\right), \mathbb{Z}\right)
$$

on the rational representation ring of $\Sigma_{n}$.

Definition 2.6. Let the group homomorphism $\phi \in \operatorname{Rep}_{\mathbb{Q}}\left(\Sigma_{n}\right)_{*}:=\operatorname{hom}_{\mathbb{Z}}\left(\operatorname{Rep}_{\mathbb{Q}}\left(\Sigma_{n}\right), \mathbb{Z}\right)$ be given. Then we define the operation $\phi_{X}: o b(A(X)) \rightarrow \bar{K}_{0}\left(A\left(X^{(n)}\right)\right)$ as follows:

$$
\begin{aligned}
& \phi_{X}: o b(A(X)) \stackrel{(-)^{\otimes n}}{\longrightarrow} \bar{K}_{0}\left(A_{\Sigma_{n}}\left(X^{n}\right)\right) \stackrel{p_{n *}}{\longrightarrow} \bar{K}_{0}\left(A_{\Sigma_{n}}\left(X^{(n)}\right)\right) \\
& \simeq \bar{K}_{0}\left(A\left(X^{(n)}\right)\right) \otimes_{\mathbb{Z}} \operatorname{Rep}_{\mathbb{Q}}\left(\Sigma_{n}\right) \stackrel{i d \otimes \phi}{\longrightarrow} \bar{K}_{0}\left(A\left(X^{(n)}\right) \otimes \mathbb{Z} \simeq \bar{K}_{0}\left(A\left(X^{(n)}\right)\right) .\right.
\end{aligned}
$$

Remark 2.7. Note that $\phi_{X}(\mathcal{V})$ only depends on the isomorphism class of $\mathcal{V} \in A(X)$. In fact, one has an induced map

$$
\phi_{X}: \bar{K}_{0}(A(X)) \rightarrow \bar{K}_{0}\left(A\left(X^{(n)}\right)\right.
$$

on the level of Grothendieck groups, which factorizes over $\phi_{X}: K_{0}(A(X)) \rightarrow K_{0}\left(A\left(X^{(n)}\right)\right)$ in case $A(-)$ takes values in a $\mathbb{Q}$-linear abelian category, with $p_{n *}$ and $\otimes$ an exact functor (in both variables). This just follows from the corresponding result for

$$
(-)^{\otimes n}: \bar{K}_{0}(A(X)) \rightarrow \bar{K}_{0}\left(A_{\Sigma_{n}}\left(X^{n}\right)\right),
$$

which in this paper we only prove for the case $X=p t$ needed for our genera.

Proposition 2.8. For $X=p t$ one gets an induced self-map

$$
\phi_{p t}: \bar{K}_{0}(A(p t)) \rightarrow \bar{K}_{0}(A(p t))
$$

on the Grothendieck group $\bar{K}_{0}(A(p t))$.

Proof. Since $A(-)$ takes values in a Karoubian $\mathbb{Q}$-linear category, one has for a space $Z$ with a trivial $G$-action for subgroups $G^{\prime} \subset G \subset \Sigma_{n}$ a $\mathbb{Q}$-linear induction functor (compare e.g. [15])

$$
\operatorname{Ind}_{G^{\prime}}^{G}: A_{G^{\prime}}(Z) \rightarrow A_{G}(Z): \mathcal{V} \mapsto \operatorname{Ind}_{G^{\prime}}^{G}(\mathcal{V}):=(\mathbb{Q}[G] \otimes \mathcal{V})^{G^{\prime}}
$$

so that it agrees on the level of Grothendieck groups with the map

$$
\operatorname{Ind}_{G^{\prime}}^{G}: \bar{K}_{0}\left(A_{G^{\prime}}(Z)\right) \simeq \bar{K}_{0}(A(Z)) \otimes_{\mathbb{Z}} \operatorname{Rep}_{\mathbb{Q}}\left(G^{\prime}\right) \rightarrow \bar{K}_{0}(A(Z)) \otimes_{\mathbb{Z}} \operatorname{Rep}_{\mathbb{Q}}(G) \simeq \bar{K}_{0}\left(A_{G}(Z)\right)
$$

coming from the usual induction homomorphism $\operatorname{Ind}_{G^{\prime}}^{G}: \operatorname{Rep}_{\mathbb{Q}}\left(G^{\prime}\right) \rightarrow \operatorname{Rep}_{\mathbb{Q}}(G)$. Then one has for $\mathcal{V}, \mathcal{V}^{\prime} \in A(p t)$ an isomorphism ([14]):

$$
\left(\mathcal{V} \oplus \mathcal{V}^{\prime}\right)^{\otimes n} \simeq \oplus_{i+j=n} \operatorname{Ind} d_{\Sigma_{i} \times \Sigma_{j}}^{\Sigma_{n}}\left(\mathcal{V}^{\otimes i} \otimes \mathcal{V}^{\otimes j}\right)
$$


so that the total power map

$$
\begin{gathered}
o b(A(p t)) \rightarrow 1+\sum_{n \geq 1} \bar{K}_{0}(A(p t)) \otimes_{\mathbb{Z}} \operatorname{Rep}_{\mathbb{Q}}\left(\Sigma_{n}\right) \cdot t^{n} \subset\left(\bar{K}_{0}(A(p t)) \otimes_{\mathbb{Z}} \operatorname{Rep}_{\mathbb{Q}}(\Sigma)\right)[[t]]: \\
\mathcal{V} \mapsto 1+\sum_{n \geq 1}\left[\mathcal{V}^{\otimes n}\right] \cdot t^{n}
\end{gathered}
$$

induces a morphism of commutative semigroups from the isomorphism classes of objects in $o b(A(p t)$ with the direct sum $\oplus$ to the group of special invertible power series in

$$
1+\sum_{n \geq 1} \bar{K}_{0}(A(p t)) \otimes_{\mathbb{Z}} \operatorname{Rep}_{\mathbb{Q}}\left(\Sigma_{n}\right) \cdot t^{n}
$$

with the power series multiplication. Here

$$
\operatorname{Rep}_{\mathbb{Q}}(\Sigma):=\oplus_{n \geq 0} \operatorname{Rep}_{\mathbb{Q}}\left(\Sigma_{n}\right)
$$

is the total (commutative) representation ring (compare e.g. [25]) with the cross product

$$
\otimes: \operatorname{Rep}_{\mathbb{Q}}\left(\Sigma_{i}\right) \times \operatorname{Rep}_{\mathbb{Q}}\left(\Sigma_{j}\right) \rightarrow \operatorname{Rep}_{\mathbb{Q}}\left(\Sigma_{i+j}\right):\left(\mathcal{V}, \mathcal{V}^{\prime}\right) \mapsto \operatorname{Ind}_{\Sigma_{i} \times \Sigma_{j}}^{\Sigma_{i+j}}\left(\mathcal{V} \otimes \mathcal{V}^{\prime}\right)
$$

So the total power map induces a group homomorphism

$$
\bar{K}_{0}(A(p t)) \rightarrow 1+\sum_{n \geq 1} \bar{K}_{0}(A(p t)) \otimes_{\mathbb{Z}} \operatorname{Rep}_{\mathbb{Q}}\left(\Sigma_{n}\right) \cdot t^{n}:[\mathcal{V}] \mapsto 1+\sum_{n \geq 1}\left[\mathcal{V}^{\otimes n}\right] \cdot t^{n}
$$

whose projection onto the summand of $t^{n}$ gives us the $n$-th power map on the level of Grothendieck groups.

From the inclusion $\Sigma_{i} \times \Sigma_{j} \rightarrow \Sigma_{i+j}$ one gets homomorphisms

$$
\operatorname{Rep}_{\mathbb{Q}}\left(\Sigma_{i+j}\right) \rightarrow \operatorname{Rep}_{\mathbb{Q}}\left(\Sigma_{i} \times \Sigma_{j}\right) \simeq \operatorname{Rep}_{\mathbb{Q}}\left(\Sigma_{i}\right) \otimes \operatorname{Rep}_{\mathbb{Q}}\left(\Sigma_{j}\right)
$$

and hence by duality

$$
\operatorname{Rep}_{\mathbb{Q}}\left(\Sigma_{i}\right)_{*} \otimes \operatorname{Rep}_{\mathbb{Q}}\left(\Sigma_{j}\right)_{*} \rightarrow \operatorname{Rep}_{\mathbb{Q}}\left(\Sigma_{i+j}\right)_{*}
$$

And this turns

$$
\operatorname{Rep}_{\mathbb{Q}}(\Sigma)_{*}:=\oplus_{n \geq 0} \operatorname{Rep}_{\mathbb{Q}}\left(\Sigma_{n}\right)_{*}
$$

into a commutative graded ring ([1]). Denoting by

$$
O p\left(\bar{K}_{0}(A(p t))\right):=\operatorname{map}\left(\bar{K}_{0}(A(p t)), \bar{K}_{0}(A(p t))\right)
$$

the operation ring of self-maps of $\bar{K}_{0}(A(p t))$ (with the pointwise addition and multiplication), the group homomorphisms

$$
\operatorname{Rep}_{\mathbb{Q}}\left(\Sigma_{i}\right)_{*} \rightarrow O p\left(\bar{K}_{0}(A(p t))\right): \phi \mapsto \phi_{p t}
$$

extend additively to a ring homomorphism

$$
\operatorname{Rep}_{\mathbb{Q}}(\Sigma)_{*} \rightarrow O p\left(\bar{K}_{0}(A(p t))\right)
$$

Note that any element $g \in \Sigma_{n}$ induces a character $\phi:=\operatorname{tr}(g): \operatorname{Rep}_{\mathbb{Q}}\left(\Sigma_{n}\right) \rightarrow \mathbb{Z}$ and therefore an operation on $\bar{K}_{0}(A(p t))$ by taking the trace of $g$ in the corresponding 
representation. And $\operatorname{tr}(g)$ depends of course only on the conjugacy class of $g \in \Sigma_{n}$. The most important operations are the following (compare [1]):

$\sigma$ : The homomorphisms $\sigma_{n}:=\frac{1}{n !} \cdot \sum_{g \in \Sigma_{n}} \operatorname{tr}(g): \operatorname{Rep}_{\mathbb{Q}}\left(\Sigma_{n}\right) \rightarrow \mathbb{Z}$ corresponds to the $n$-th symmetric power operation $[\mathcal{V}] \mapsto\left[\left(\mathcal{V}^{\otimes n}\right)^{\Sigma_{n}}\right]$.

$\lambda$ : The homomorphisms $\lambda_{n}:=\frac{1}{n !} \cdot \sum_{g \in \Sigma_{n}}(-1)^{\operatorname{sign}(g)} \cdot \operatorname{tr}(g): \operatorname{Rep}_{\mathbb{Q}}\left(\Sigma_{n}\right) \rightarrow \mathbb{Z}$ corresponds to the $n$-th antisymmetric power operation $[\mathcal{V}] \mapsto\left[\left(\mathcal{V}^{\otimes n}\right)^{s i g n-\Sigma_{n}}\right]$.

$\Psi:$ If $g \in \Sigma_{n}$ is an $n$-cycle, then $\operatorname{tr}(g)$ corresponds by [1] [cor.1.8] to the $n$-th Adams operation $\Psi_{n}: \bar{K}_{0}(A(p t)) \rightarrow \bar{K}_{0}(A(p t))$ associated to the pre-lambda structure $\sigma_{t}$ (or $\lambda_{t}$ depending on the chosen conventions).

Finally $\operatorname{Rep}_{\mathbb{Q}}(\Sigma)_{*}$ is a polynomial ring on the generators $\left\{\sigma_{n} \mid n \in \mathbb{N}\right\}$ (or $\left\{\lambda_{n} \mid n \in \mathbb{N}\right\}$ ), and $\operatorname{Rep}_{\mathbb{Q}}(\Sigma)_{*} \otimes_{\mathbb{Z}} \mathbb{Q}$ is a polynomial ring on the generators $\left\{\Psi_{n} \mid n \in \mathbb{N}\right\}([1])$.

The important point for us is the fact that we have corresponding "coefficients" for $\mathcal{M} \in A(X)$ on the symmetric products:

Definition 2.9. The group homomorphisms $\phi \in \operatorname{Rep}_{\mathbb{Q}}(\Sigma)_{*}$ discussed above induce the following "coefficients" on the symmetric products $X^{(n)}$ :

$\sigma: \mathcal{M}^{(n)}=\left(\mathcal{M}^{\bigotimes n}\right)^{\Sigma_{n}} \in A\left(X^{(n)}\right)$.

$\lambda: \mathcal{M}^{\{n\}}=\left(\mathcal{M}^{\bigotimes n}\right)^{s i g n-\Sigma_{n}} \in A\left(X^{(n)}\right)$.

$\Psi: \Psi_{n}(\mathcal{M}):=\phi_{X}(\mathcal{M}) \in \bar{K}_{0}\left(A\left(X^{(n)}\right)\right)$, with $\phi:=\operatorname{tr}(g)$ for $g \in \Sigma_{n}$ an n-cycle.

The (anti)symmetric powers $\mathcal{M}^{(n)}, \mathcal{M}^{\{n\}}$ already appeared before. And the Adams operations are needed for the formulation of our last result of this section.

Theorem 2.10. Consider the category space of complex quasi-projective varietes. Assume that the Künneth morphism for the pseudofunctor $(-)_{*}$ is an isomorphism for all constant morphisms $k: X \rightarrow$ t. Then for any $\mathcal{M} \in$ ob $(A(X))$ we obtain the following abstract generating series in $\bar{K}_{0}(A(p t)) \otimes_{\mathbb{Z}} \mathbb{Q}[[t]]$ :

$$
1+\sum_{n \geq 1}\left(\left[k_{*} \mathcal{M}\right]^{\otimes n}\right)^{\Sigma_{n}} \cdot t^{n}=\exp \left(\sum_{r \geq 1} \Psi_{r}\left(\left[k_{*} \mathcal{M}\right]\right) \cdot \frac{t^{r}}{r}\right)
$$

and

$$
1+\sum_{n \geq 1}\left(\left[k_{*} \mathcal{M}\right]^{\otimes n}\right)^{\operatorname{sign}-\Sigma_{n}} \cdot t^{n}=\exp \left(-\sum_{r \geq 1} \Psi_{r}\left(\left[k_{*} \mathcal{M}\right]\right) \cdot \frac{(-t)^{r}}{r}\right) .
$$

Moreover we have the following isomorphisms in $\bar{K}_{0}(A(p t))$ :

$$
\begin{gathered}
\left(\left[k_{*} \mathcal{M}\right]^{\otimes n}\right)^{\Sigma_{n}} \simeq k_{*}\left[\mathcal{M}^{(n)}\right], \quad\left(\left[k_{*} \mathcal{M}\right]^{\otimes n}\right)^{\text {sign- } \Sigma_{n}} \simeq k_{*}\left[\mathcal{M}^{\{n\}}\right] \\
\text { and } \Psi_{r}\left(\left[k_{*} \mathcal{M}\right]\right) \simeq k_{*}\left(\Psi_{r}(\mathcal{M})\right)
\end{gathered}
$$

Here the isomorphisms in the last part of the theorem follow from the fact that the decomposition (46) of the Grothendieck group of $\Sigma_{n}$-equivariant objects is functorial under maps between spaces with trivial $\Sigma_{n}$-action, e.g. the constant map $k: X^{(n)} \rightarrow p t$. 
Formula (50) is a consequence of (44) by using the pre-lambda structure from (35). Formula (51) follows then by using the opposite pre-lambda structure (41).

\section{EqUiVARIANT GENERA AND TRACE HOMOMORPHISMS}

In this section we explain a different proof of Corollary 1.2 and Theorem 2.10 , based on equivariant genera and (abstract) trace homomorphisms. This proof follows a standard strategy for obtaining generating series formulae (e.g., it was used by Moonen or Zagier for proving their results), and it is the one that will be generalized in a forthcoming paper to corresponding results for the Hirzebruch classes of Brasselet-Schürmann-Yokura [10].

Since the symmetric group $\Sigma_{n}$ acts graded anti-symmetrically on $H_{(c)}^{*}\left(X^{n}, \mathcal{M}^{\bigotimes n}\right) \simeq$ $H_{(c)}^{*}(X, \mathcal{M})^{\otimes n}$, it makes sense to take traces of this action. So we can define equivariant Hodge genera by (compare [11]):

$$
\chi_{-y}^{(c)}\left(X^{n}, \mathcal{M}^{\otimes n} ; \sigma\right):=\sum_{i, p}(-1)^{i} \operatorname{trace}\left(\sigma \mid \operatorname{Gr}_{F}^{p}\left(H_{(c)}^{i}\left(X^{n}, \mathcal{M}^{\otimes n}\right) \otimes \mathbb{C}\right)\right) \cdot y^{p} .
$$

In order to simplify the expressions below, in what follows we leave out the reference to $\mathbb{C}$-coefficients when taking the Hodge filtrations into account.

Lemma 3.1. For any $n \geq 0$,

$$
\chi_{-y}^{(c)}\left(X^{(n)}, \mathcal{M}^{(n)}\right)=\frac{1}{n !} \sum_{\sigma \in \Sigma_{n}} \chi_{-y}^{(c)}\left(X^{n}, \mathcal{M}^{\bigotimes n} ; \sigma\right)
$$

Proof.

$$
\begin{aligned}
\chi_{-y}^{(c)}\left(X^{(n)}, \mathcal{M}^{(n)}\right) & =\sum_{i, p}(-1)^{i} \operatorname{dim}_{\mathbb{C}} \operatorname{Gr}_{F}^{p} H_{(c)}^{i}\left(X^{(n)}, \mathcal{M}^{(n)}\right) \cdot y^{p} \\
& \stackrel{(*)}{=} \sum_{i, p}(-1)^{i} \operatorname{dim}_{\mathbb{C}} \operatorname{Gr}_{F}^{p}\left(H_{(c)}^{i}\left(X^{n}, \mathcal{M}^{\bigotimes n}\right)^{\Sigma_{n}}\right) \cdot y^{p} \\
& =\sum_{i, p}(-1)^{i} \operatorname{dim}_{\mathbb{C}}\left(\operatorname{Gr}_{F}^{p} H_{(c)}^{i}\left(X^{n}, \mathcal{M}^{\bigotimes n}\right)\right)^{\Sigma_{n}} \cdot y^{p} \\
& =\sum_{i, p}(-1)^{i}\left(\frac{1}{n !} \sum_{\sigma \in \Sigma_{n}} \operatorname{trace}\left(\sigma \mid \operatorname{Gr}_{F}^{p} H_{(c)}^{i}\left(X^{n}, \mathcal{M}^{\bigotimes n}\right)\right)\right) \cdot y^{p} \\
& =\frac{1}{n !} \sum_{\sigma \in \Sigma_{n}}\left(\sum_{i, p}(-1)^{i} \operatorname{trace}\left(\sigma \mid \operatorname{Gr}_{F}^{p} H_{(c)}^{i}\left(X^{n}, \mathcal{M}^{\bigotimes n}\right)\right) \cdot y^{p}\right),
\end{aligned}
$$

where the equality labeled $(*)$ follows from the fact that (9) is an isomorphism of graded groups of mixed Hodge structures, identifying $H_{(c)}^{*}\left(X^{n}, \mathcal{M}^{\otimes n}\right)^{\Sigma_{n}}$ with a sub-mixed Hodge structure of $H_{(c)}^{*}\left(X^{n}, \mathcal{M}^{\bigotimes n}\right)$. 
Lemma 3.2. If $\sigma \in \Sigma_{n}$ has cycle-type $\left(k_{1}, k_{2}, \cdots, k_{n}\right)$, that is, $k_{r}$ is the number of length $r$ cycles in $\sigma$ and $\sum_{r=1}^{n} k_{r} \cdot r=n$, then

$$
\chi_{-y}^{(c)}\left(X^{n}, \mathcal{M}^{\otimes n} ; \sigma\right)=\prod_{r=1}^{n} \chi_{-y}^{(c)}\left(X^{r}, \mathcal{M}^{\otimes r} ; \sigma_{r}\right)^{k_{r}},
$$

with $\sigma_{r}=(12 \cdots r)$ an $r$-cycle.

Proof. This is immediate from properties of the trace and from the Künneth isomorphism

$$
H_{(c)}^{*}\left(X^{n}, \mathcal{M}^{\otimes n}\right) \simeq \otimes_{r} H_{(c)}^{*}\left(X^{r}, \mathcal{M}^{\otimes r}\right)^{\otimes k_{r}},
$$

where the tensor product of the right-hand side term runs over the positive integers $r$ appearing in the partition condition $\sum_{r=1}^{n} k_{r} \cdot r=n$. Note that the isomorphism (55) is compatible with the mixed Hodge structures.

Therefore it suffices to understand the equivariant Hodge genera $\chi_{-y}^{(c)}\left(X^{r}, \mathcal{M}^{\bigotimes r} ; \sigma_{r}\right)$ for all $r$-cycles in $\Sigma_{r}$. By the considerations of section 2.2 this corresponds to the Adams operation $\Psi_{r}$ on $\mathbb{Z}\left[y^{ \pm 1}\right]$. So by Example 2.5 we get

$$
\chi_{-y}^{(c)}\left(X^{r}, \mathcal{M}^{\otimes r} ; \sigma_{r}\right)=\Psi_{r}\left(\chi_{-y}^{(c)}(X, \mathcal{M})\right)=\chi_{-y^{r}}^{(c)}(X, \mathcal{M}) .
$$

The same reasoning applies to the abstract context of Theorem 2.10 by using "abstract trace homomorphisms", i.e. the operations $\phi_{p t} \in O p\left(\bar{K}_{0}(A(p t))\right)$ of Proposition [2.8 coming from a character $\phi=\operatorname{tr}(g): \operatorname{Rep}_{\mathbb{Q}}\left(\Sigma_{n}\right) \rightarrow \mathbb{Z}$. Indeed, Lemma 3.1 corresponds to taking the projector onto the symmetric part

$$
\sigma_{n}\left(\left[k_{*} \mathcal{M}\right]\right)=\left(\left[k_{*} \mathcal{M}\right]^{\otimes n}\right)^{\Sigma_{n}}=\frac{1}{n !} \sum_{\sigma \in \Sigma_{n}} \operatorname{tr}(\sigma)_{p t}\left(\left[k_{*} \mathcal{M}\right]\right) .
$$

Lemma 3.2 corresponds to the fact that the map (49)

$$
\operatorname{Rep}_{\mathbb{Q}}(\Sigma)_{*} \rightarrow O p\left(\bar{K}_{0}(A(p t))\right)
$$

is a ring homomorphism. So for $\sigma$ of cycle-type $\left(k_{1}, k_{2}, \cdots, k_{n}\right)$ one has

$$
\operatorname{tr}(\sigma)_{p t}\left(\left[k_{*} \mathcal{M}\right]\right)=\prod_{r=1}^{n}\left(\operatorname{tr}\left(\sigma_{r}\right)_{p t}\left(\left[k_{*} \mathcal{M}\right]\right)\right)^{k_{r}} \in \bar{K}_{0}(A(p t))
$$

Finally, equation (56) just corresponds to the abstract Adams operation on $\bar{K}_{0}(A(p t))$ :

$$
\operatorname{tr}\left(\sigma_{r}\right)_{p t}\left(\left[k_{*} \mathcal{M}\right]\right)=\Psi_{r}\left(\left[k_{*} \mathcal{M}\right]\right) .
$$

Remark 3.3. This is not just an analogy, as one can go from the abstract formalism back to the concret trace formulae for $g \in \Sigma_{n}$ by using the following ring homomorphisms on 
the corresponding Grothendieck group of $\Sigma_{n}$-equivariant objects in $A(p t):=D^{b} M H M(p t)$ :

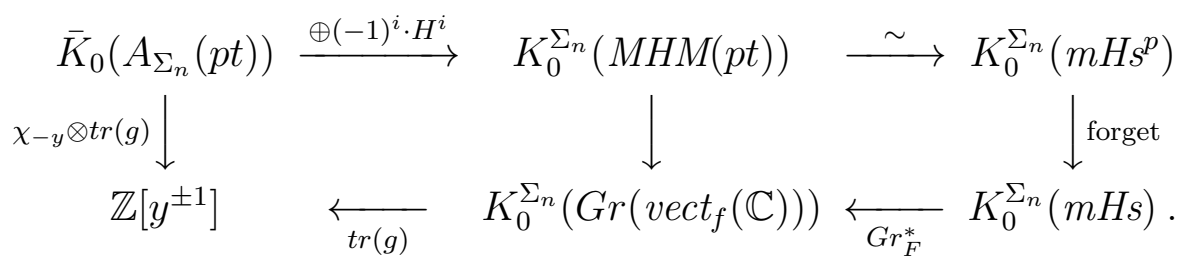

Here the first vertical arrow is induced from the decomposition (46) of the equivariant Grothendieck group:

$$
\bar{K}_{0}\left(A_{\Sigma_{n}}(p t)\right) \simeq \bar{K}_{0}(A(p t)) \otimes_{\mathbb{Z}} \operatorname{Rep}_{\mathbb{Q}}\left(\Sigma_{n}\right) .
$$

If we apply the composed homomorphism to $\left[k_{*} \mathcal{M}\right] \in \bar{K}_{0}\left(A_{\Sigma_{n}}(p t)\right)$ we get back the equivariant Hodge genus $\chi_{-y}^{(c)}\left(X^{n}, \mathcal{M}^{\bigotimes n} ; \sigma\right)$. Here the abstract pushforward $k_{*}$ stands for either $k_{*}$ or $k_{!}$in the context of mixed Hodge modules.

Putting everything together we now obtain Corollary 1.2 as follows. For a given partition $\Pi=\left(k_{1}, k_{2}, \cdots, k_{n}\right)$ of $n$, i.e., $n=\sum_{r} k_{r} \cdot r$, denote by $N_{\Pi}$ the number of elements $\sigma \in \Sigma_{n}$ of cycle-type $\Pi$. It is then easy to see that

$$
N_{\Pi}=\frac{n !}{k_{1} ! k_{2} ! \cdots 1^{k_{1}} 2^{k_{2}} \cdots} .
$$

It follows that

$$
\begin{aligned}
& \sum_{n} \chi_{-y}^{(c)}\left(X^{(n)}, \mathcal{M}^{(n)}\right) \cdot t^{n} \stackrel{553}{=} \sum_{n} t^{n} \cdot \frac{1}{n !} \sum_{\sigma \in \Sigma_{n}} \chi_{-y}^{(c)}\left(X^{n}, \mathcal{M}^{\bigotimes n} ; \sigma\right) \\
& \stackrel{\text { (54) }}{=} \sum_{n} \frac{t^{n}}{n !} \cdot \sum_{\Pi=\left(k_{1}, k_{2}, \cdots k_{n}\right)} N_{\Pi} \prod_{r=1}^{n} \chi_{-y}^{(c)}\left(X^{r}, \mathcal{M}^{\otimes r} ; \sigma_{r}\right)^{k_{r}} \\
& =\sum_{n} \sum_{\Pi=\left(k_{1}, k_{2}, \cdots k_{n}\right)} \frac{t^{k_{1} \cdot 1+k_{2} \cdot 2+\cdots}}{k_{1} ! k_{2} ! \cdots 1^{k_{1}} 2^{k_{2}} \cdots} \prod_{r=1}^{n} \chi_{-y}^{(c)}\left(X^{r}, \mathcal{M}^{\otimes r} ; \sigma_{r}\right)^{k_{r}} \\
& =\sum_{n} \sum_{\Pi=\left(k_{1}, k_{2}, \cdots k_{n}\right)} \prod_{r=1}^{n} \frac{t^{k_{r} \cdot r}}{k_{r} ! r^{k_{r}}} \cdot \chi_{-y}^{(c)}\left(X^{r}, \mathcal{M}^{\otimes r} ; \sigma_{r}\right)^{k_{r}} \\
& =\prod_{r=1}^{\infty}\left(\sum_{k_{r}=0}^{\infty} \frac{t^{k_{r} \cdot r}}{k_{r} ! r^{k_{r}}} \cdot \chi_{-y}^{(c)}\left(X^{r}, \mathcal{M}^{\otimes r} ; \sigma_{r}\right)^{k_{r}}\right) \\
& =\prod_{r=1}^{\infty} \exp \left(\frac{t^{r}}{r} \cdot \chi_{-y}^{(c)}\left(X^{r}, \mathcal{M}^{\otimes r} ; \sigma_{r}\right)\right) \\
& \stackrel{\text { 566) }}{=} \exp \left(\sum_{r=1}^{\infty} \frac{t^{r}}{r} \cdot \Psi_{r}\left(\chi_{-y}^{(c)}(X, \mathcal{M})\right)\right) \text {. }
\end{aligned}
$$


Exactly the same arguments yield a proof of Theorem 2.10 by substituting $\left[k_{*} \mathcal{M}\right]$ for $\chi_{-y}^{(c)}(X, \mathcal{M}), \sigma_{n}\left(\left[k_{*} \mathcal{M}\right]\right)$ for $\chi_{-y}^{(c)}\left(X^{(n)}, \mathcal{M}^{(n)}\right)$, and finally, $\operatorname{tr}(g)_{p t}\left(\left[k_{*} \mathcal{M}\right]\right)$ in place of $\chi_{-y}^{(c)}\left(X^{n}, \mathcal{M}^{\otimes n} ; g\right)$.

\section{Appendix A: Exterior products And EQUivariant obJECTS}

In this appendix we collect in an abstract form the categorical notions needed to formulate and prove our main results, about the relation between exterior products and equivariant objects. Here we work over a category space of spaces, which for us are the quasi-projective complex algebraic varieties $X$. But the same arguments also apply to other kinds of categories of "spaces", e.g., (compact) topological or complex analytic spaces.

4.1. Cofibered Categories. Let space be a (small) category with finite products $\times$ and terminal object $p t$ (corresponding to the empty product). The universal property of the product $\times$ makes space into a symmetric monoidal category [8] [sec.6.1], i.e., for $X, Y, Z \in o b($ space $)$ there are functorial isomorphisms

(associativity)

(units)

(symmetry)

$$
\begin{gathered}
a:(X \times Y) \times Z \stackrel{\sim}{\rightarrow} X \times(Y \times Z), \\
l: p t \times X \stackrel{\sim}{\rightarrow} X \text { and } r: X \times p t \stackrel{\sim}{\rightarrow} X, \\
s: X \times Y \stackrel{\sim}{\rightarrow} Y \times X,
\end{gathered}
$$

such that $s^{2}=i d$ and the following diagrams commute:
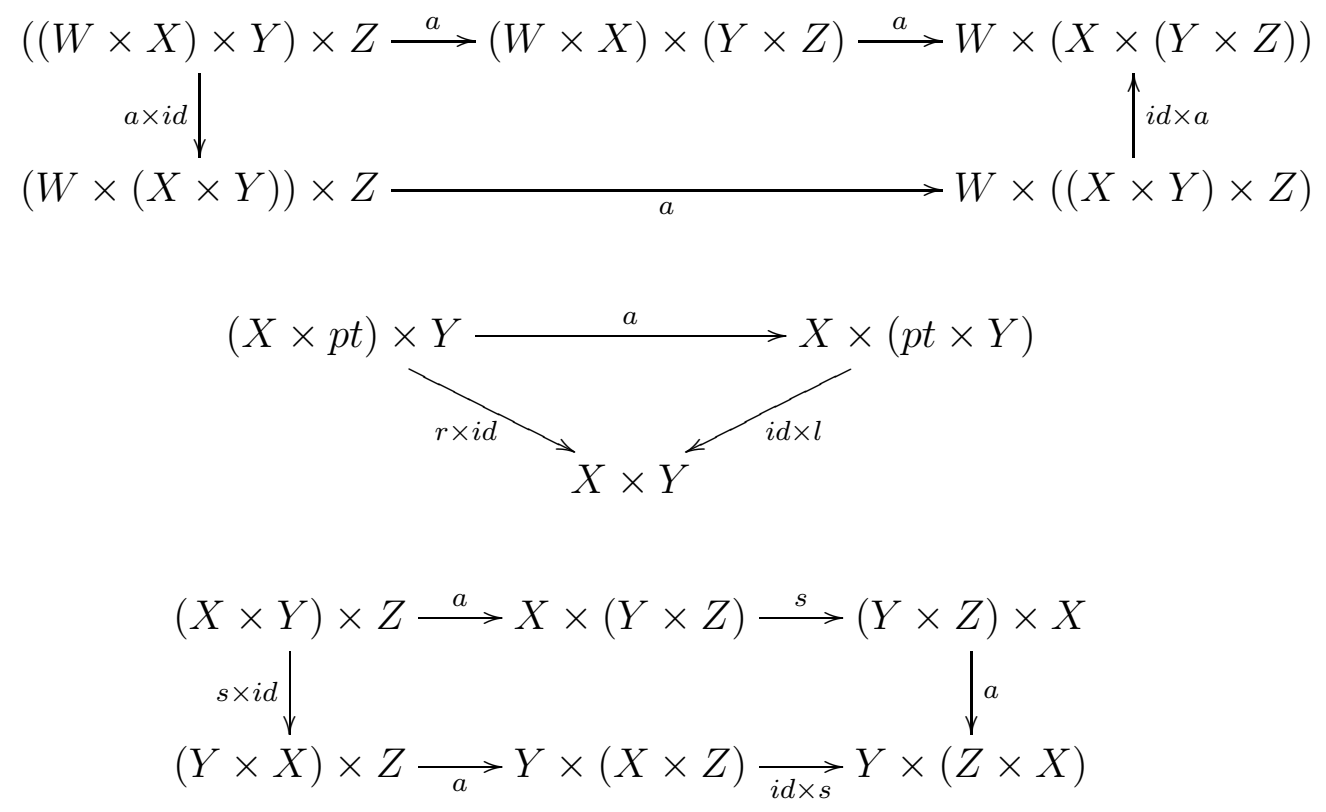
and

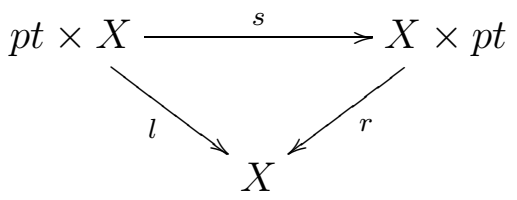

Let us define inductively $X^{0}:=p t, X^{1}:=X$ and $X^{n+1}:=X \times X^{n}$ so that a morphism $f: X \rightarrow Y$ induces $f^{n}: X^{n} \rightarrow Y^{n}$. Then the above constraints for $\times$ imply that the definition of $X^{n}$ does not depend (up to canonical isomorphisms) on the chosen order of brackets. Moreover $X^{n}$ gets a canonical induced (left) $\Sigma_{n}$-action such $f^{n}$ is an equivariant morphism.

In addition, we fix a covariant pseudofunctor $(-)_{*}$ on space (compare e.g. [41] [ch.3] or [27][Part II, Ch.1]), i.e., a category $A(X)$ for all $X \in o b($ space) and (push down) functors $f_{*}: A(X) \rightarrow A(Y)$ for all morphisms $f: X \rightarrow Y$ in space, together with natural isomorphisms

$$
e: i d_{A(X)} \stackrel{\sim}{\rightarrow} i d_{X *}
$$

and

$$
c:(g f)_{*} \stackrel{\sim}{\rightarrow} g_{*} f_{*}
$$

for all composable pairs of morphisms $f, g$ in space, such that the following conditions are satisfied:

(PS1) For any morphism $f: X \rightarrow Y$ the map

$$
f_{*}\left(i d_{A(X)}\right)=f_{*}=\left(f \circ i d_{X}\right)_{*} \stackrel{c}{\rightarrow} f_{*}\left(i d_{X *}\right)
$$

agrees with $f_{*}(e)$.

(PS2) For any morphism $f: X \rightarrow Y$ the map

$$
i d_{A(Y)}\left(f_{*}\right)=f_{*}=\left(i d_{Y} \circ f\right)_{*} \stackrel{c}{\rightarrow} i d_{Y *}\left(f_{*}\right)
$$

agrees with $e\left(f_{*}\right)$.

(PS3) For any triple of composable morphisms

$$
X \stackrel{f}{\longrightarrow} Y \stackrel{g}{\longrightarrow} Z \stackrel{h}{\longrightarrow} W,
$$

the following diagram commutes:

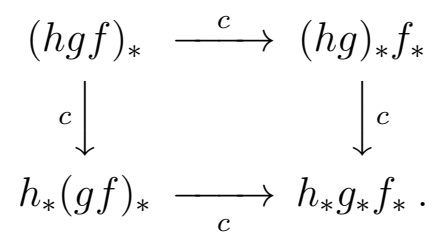

Note that in many cases (e.g. in all our applications) $e$ is just the identity $i d_{A(X)}=i d_{X *}$. The above conditions allow to make the pairs $(X, M)$ with $X \in$ ob(space) and $M \in A(X)$ into a category $A$ space, were a morphism $(f, \phi):(X, M) \rightarrow(Y, N)$ is given by a 
morphism $f: X \rightarrow Y$ in space together with a morphism $\phi: f_{*}(M) \rightarrow N$ in $A(Y)$. The composition of morphisms

$$
(X, M) \stackrel{(f, \phi)}{\longrightarrow}(Y, N) \stackrel{(g, \psi)}{\longrightarrow}(Z, O)
$$

is defined by

$$
(g f)_{*} M \stackrel{c}{\longrightarrow} g_{*} f_{*} M \stackrel{g_{*}(\phi)}{\longrightarrow} g_{*} N \stackrel{\psi}{\longrightarrow} O .
$$

Then the associativity of the composition follows from (PS3) above, whereas $\left(i d, e^{-1}\right)$ becomes the identity arrow by (PS1) and (PS2). The projection $p: A /$ space $\rightarrow$ space onto the first component defines a functor making $A$ /space into a cofibered (or sometimes also called opfibered) category over space, with $A(X)$ isomorphic to the fiber over $X$. Here $\left(i d_{X}, \tilde{\phi}\right):(X, M) \rightarrow(X, N)$ corresponds to $\phi:=\tilde{\phi} \circ e: M \rightarrow i d_{X *}(M) \rightarrow N$. In particular, any morphism $(f, \phi):(X, M) \rightarrow(Y, N)$ in $A /$ space can be decomposed as $(f, \phi)=\left(i d_{Y}, \tilde{\phi}\right) \circ\left(f, i d_{f_{*} M}\right)$.

Sometimes it is more natural to work with the opposite arrows in $A(X)$ and to think of $(-)_{*}$ as a pseudofunctor with "values" in the opposite category $A^{o p}(-)$. Then the pairs $(X, M)$ with $X \in o b($ space $)$ and $M \in A(X)$ become a category $A^{o p} /$ space, were a morphism $(f, \phi):(X, M) \rightarrow(Y, N)$ is given by a morphism $f: X \rightarrow Y$ in space together with a morphism $\phi: N \rightarrow f_{*}(M)$ in $A(Y)$. The composition of morphisms

$$
(X, M) \stackrel{(f, \phi)}{\longrightarrow}(Y, N) \stackrel{(g, \psi)}{\longrightarrow}(Z, O)
$$

is then defined by

$$
O \stackrel{\psi}{\longrightarrow} g_{*} N \stackrel{g_{*}(\phi)}{\longrightarrow} g_{*} f_{*} M \stackrel{c^{-1}}{\longrightarrow}(g f)_{*} M
$$

with $(i d, e)$ the identity arrow.

4.2. Equivariant objects. Suppose that the group $G$, with unit 1, acts (from the left) on $X$, i.e. we have a group homomorphism $\phi: G \rightarrow A u t_{\text {space }}(X)$. Then a $G$-equivariant object $M \in A(X)$ is by definition given by a family of isomorphisms

$$
\tilde{\phi}_{g}: g_{*} M \rightarrow M \quad(g \in G)
$$

such that

$$
\tilde{\phi}_{1}=e^{-1} \text { and } \tilde{\phi}_{g f}=\tilde{\phi}_{g} \circ g_{*}\left(\tilde{\phi}_{f}\right) \circ c \text { for all } f, g \in G
$$

This just means that $(X, M) \in o b(A /$ space $)$ has a $G$-action given by a group homomorphism $\tilde{\phi}: G \rightarrow A u t_{A / \text { space }}((X, M))$. With the obvious morphisms, this defines the category $A_{G}(X)$ of $G$-equivariant objects in $A(X)$. If $G$ acts trivially on $X$, i.e., $g=i d_{X}$ for all $g \in G$, then this corresponds to an action of $G$ on $M$ in $A(X)$, i.e., a group homomorphism $\phi: G \rightarrow A_{A(X)}(M)$. For a $G$-equivariant morphism $f: X \rightarrow Y$ of $G$-spaces one gets an induced functorial push down $f_{*}: A_{G}(X) \rightarrow A_{G}(Y)$, defining a 
covariant pseudofunctor on the category $G$ - space. If we prefer to work with $A^{o p}$, we can use the isomorphisms

$$
\tilde{\psi}_{g}:=\tilde{\phi}_{g}^{-1}: M \rightarrow g_{*} M \quad(g \in G)
$$

such that

$$
\tilde{\psi}_{1}=e \quad \text { and } \quad \tilde{\psi}_{g f}=c^{-1} \circ g_{*}\left(\tilde{\psi}_{f}\right) \circ \tilde{\psi}_{g} \quad \text { for all } \quad f, g \in G .
$$

Then a $G$-equivariant object $M \in A_{G}(X)$ corresponds to a $G$-action on $(X, M)$ in $o b\left(A^{o p} /\right.$ space $)$ given by a group homomorphism $\tilde{\psi}: G \rightarrow A u t_{A^{o p} / \text { space }}((X, M))$. If $G$ acts trivially on $X$, then this corresponds to an action of $G$ on $M$ in $A(X)$, i.e. a group homomorphism $\psi: G \rightarrow A u t_{A(X)}(M)$ given by isomorphisms

$$
\psi_{g}:=\phi_{g}^{-1}: M \rightarrow M \quad(g \in G)
$$

And this is the version needed in this paper for $\Sigma_{n}$-equivariant objects on symmetric products $X^{(n)}$.

Assume now that $A /$ space (or $A^{o p} /$ space) has the structure of a symmetric monoidal category such that the projection $p: A /$ space $\rightarrow$ space (or $p: A^{o p} /$ space $\rightarrow$ space) onto the first component is a strict monoidal functor. So we have a functorial "product" or "pairing"

$$
(X, M) \otimes(Y, N)=(X \times Y, M \otimes N)
$$

together with associativity, unit and symmetry isomorphisms $a, l, r, s$ in $A /$ space (or $A^{o p} /$ space) satisfying $s^{2}=i d$, (57), (58), (59) and (50). If we identify $p t^{n}$ with $p t$ by the natural projection isomorphism $p_{n *}: p t^{n} \stackrel{\sim}{\rightarrow} p t$, then $A(p t)$ becomes a symmetric monoidal category with unit $1_{p t} \in A(p t)$ and product

$$
M \otimes M^{\prime}:=p_{2 *}\left(M \otimes M^{\prime}\right) .
$$

Similarly one gets for $M \in A(X)$ the $\Sigma_{n}$-equivariant object $M^{\bigotimes n} \in A\left(X^{n}\right)$ corresponding to the $\Sigma_{n}$-equivariant object $(X, M)^{n}$ in $A /$ space (or in $A^{\text {op }} /$ space)!

4.3. Künneth morphisms. If one looks at the morphisms $\left(f, i d_{f_{*} M}\right):(X, M) \rightarrow$ $\left(Y, f_{*} M\right)$ and $\left(f^{\prime}, i d_{f_{*}^{\prime} M^{\prime}}\right):\left(X^{\prime}, M^{\prime}\right) \rightarrow\left(Y^{\prime}, f_{*}^{\prime} M^{\prime}\right)$ in $A /$ space (or $A^{o p} /$ space), then the functoriality of $\otimes$ gives by

$$
\left(f, i d_{f_{*} M}\right) \otimes\left(f^{\prime}, i d_{f_{*}^{\prime} M^{\prime}}\right)=:\left(f \times f^{\prime}, \text { Kue }\right)
$$

a Künneth morphism in $A\left(Y \times Y^{\prime}\right)$ :

Kue $:\left(f \times f^{\prime}\right)_{*}\left(M \otimes M^{\prime}\right) \rightarrow f_{*} M \otimes f_{*}^{\prime} M^{\prime} \quad$ or $\quad$ Kue $: f_{*} M \otimes f_{*}^{\prime} M^{\prime} \rightarrow\left(f \times f^{\prime}\right)_{*}\left(M \otimes M^{\prime}\right)$, functorial in $M \in o b(A(X))$ and $M^{\prime} \in o b\left(A\left(X^{\prime}\right)\right)$. In many examples, Kue is an isomorphism and one can easily switch between both viewpoints. In all our applications, one has a natural Künneth morphism

$$
\text { Kue }: f_{*} M \otimes f_{*}^{\prime} M^{\prime} \rightarrow\left(f \times f^{\prime}\right)_{*}\left(M \otimes M^{\prime}\right)
$$


so that we have to work with a pseudofunctor with "values" in the opposite category $A^{o p}(-)$. So we only spell out for this case where such a symmetric monoidal structure on $A^{\text {op }} /$ space (over $($ space,$\times)$ ) really comes from. First of all the "pairing" or bifunctor

$$
\otimes: A^{o p} / \text { space } \times A^{o p} / \text { space } \rightarrow A^{o p} / \text { space }
$$

corresponds to

(p1) An exterior product $\otimes: A(X) \times A\left(X^{\prime}\right) \rightarrow A\left(X \times X^{\prime}\right) ;\left(M, M^{\prime}\right) \mapsto M \otimes M^{\prime}$ bifunctorial in $M, M^{\prime}$.

(p2) A covariant pseudofunctor $(-)_{*}$ with "values" in the opposite category $A^{o p}(-)$.

(p3) For all morphism $f: X \rightarrow Y$ and $f^{\prime}: X^{\prime} \rightarrow Y^{\prime}$ in space, a Künneth morphism in $A\left(Y \times Y^{\prime}\right)$ :

$$
\text { Kue }: f_{*} M \otimes f_{*}^{\prime} M^{\prime} \rightarrow\left(f \times f^{\prime}\right)_{*}\left(M \otimes M^{\prime}\right),
$$
functorial in $M \in o b(A(X))$ and $M^{\prime} \in o b\left(A\left(X^{\prime}\right)\right)$.

(p4) These satisfy for $f, f^{\prime}, M, M^{\prime}$ as in (p3) and morphisms $g: Y \rightarrow Z, g^{\prime}: Y^{\prime} \rightarrow Z^{\prime}$ in space the compability

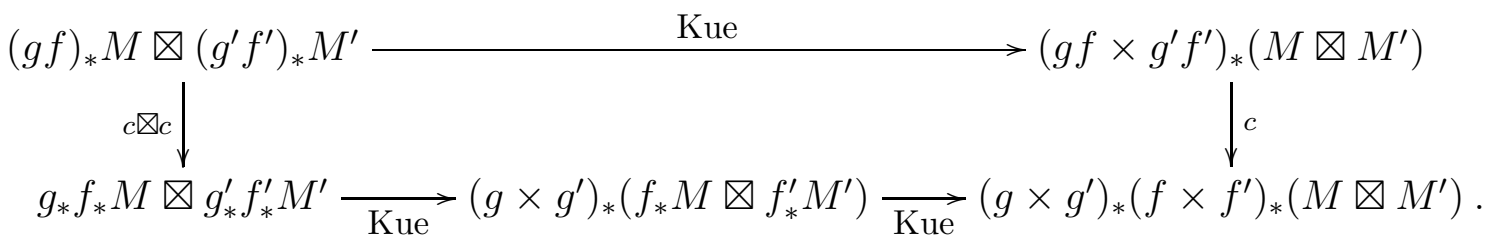

Then the associativity, unit and symmetry isomorphisms $a, l, r, s$ in $A^{\text {op }} /$ space are given by isomorphisms functorial in $M \in A(X), M^{\prime} \in A(Y)$ and $M^{\prime \prime} \in A(Z)$ :

$$
\begin{gathered}
M \otimes\left(M^{\prime} \otimes M^{\prime \prime}\right) \stackrel{\sim}{\rightarrow} a_{*}\left(\left(M \otimes M^{\prime}\right) \otimes M^{\prime \prime}\right), \\
M \stackrel{\sim}{\rightarrow} l_{*}\left(1_{p t} \otimes M\right) \quad \text { and } \quad M \stackrel{\sim}{\rightarrow} r_{*}\left(M \otimes 1_{p t}\right), \\
M \otimes M^{\prime} \stackrel{\sim}{\rightarrow} s_{*}\left(M^{\prime} \otimes M\right) .
\end{gathered}
$$

And these have to be compatible with the Künneth morphism (65) according to the functoriality of $\otimes$ given by (64). Note that for the definition of $\otimes$, we only have to work over the symmetric monoidal subcategory space ${ }^{i s o}$ of space, with the same objects and only all isomorphisms as morphisms. In this case one can easily switch between covariant and contravariant pseudofunctors using $f^{*}:=\left(f^{-1}\right)_{*}$.

Remark 4.1. In most of our applications, we consider the case when the pseudofunctor $(-)_{*}$ takes values in $(\mathbb{Q}$-linear) additive categories so that one can look at the corresponding Grothendieck groups $\bar{K}_{0}(X)$. Then we assume that $A(X)$ is a (Q্Q-linear) additive category for all $X \in$ ob(space), with $f_{*}$ and all used isomorphisms a, l, $r, s$ (Q্-linear) additive, as well as the exterior product $\otimes$ and the Künneth morphism (65) (Q-linear) additive in both variables. So these induce also homomorphisms of the corresponding Grothendieck groups $\bar{K}_{0}(-)$. If a group $G$ acts on $X$, then also the category of $G$ equivariant objects $A_{G}(X)$ becomes a $(\mathbb{Q}$-linear) additive category. 
Finally note that the opposite fibration of $A^{\text {op }} /$ space becomes then a (discrete) fibered symmetric bimonoidal category in the sense of [17].

4.4. Examples. In most applications the exterior product $\otimes$ comes from an interior product $\otimes$ on $A(X)$ making it into a symmetric monoidal category with unit $1_{X} \in A(X)$, together with a contravariant pseudofunctor $f^{*}$ on space compatible with the symmetric monoidal structure (e.g., $f^{*} 1_{Y} \simeq 1_{X}$ and $f^{*}(-\times-) \simeq f^{*}(-) \times f^{*}(-)$ for a morphism $f: X \rightarrow Y$ in space). Using the projections

$$
X \stackrel{p}{\longleftarrow} X \times X^{\prime} \stackrel{q}{\longrightarrow} X^{\prime}
$$

one defines for $M \in A(X)$ and $M^{\prime} \in A\left(X^{\prime}\right)$ the exterior product by

$$
M \otimes M^{\prime}:=p^{*} M \otimes q^{*} M^{\prime} .
$$

Then one only needs in addition a Künneth morphism (p3) for the covariant pseudofunctor $(-)_{*}$ satisfying the compability $(\mathrm{p} 4)$. Here are different examples to get such a structure:

Example 4.2 (adjoint pair). The functors $f_{*}$ are right adjoint to $f^{*}$ so that the pseudofunctors $(-)^{*},(-)_{*}$ form an adjoint pair as in [27][part I, sec.3.6]. Here the Künneth morphism Kue for the cartesian diagram

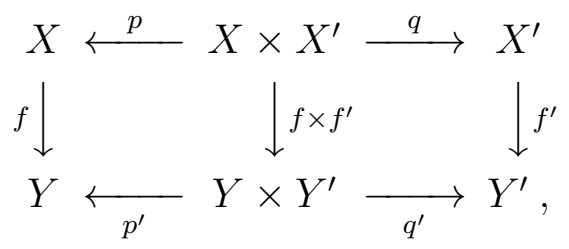

with $M \in A(X)$ and $M^{\prime} \in A\left(X^{\prime}\right)$ is induced by adjunction from the morphism

$$
\begin{aligned}
\left(f \times f^{\prime}\right)^{*}\left(f_{*} M \otimes f_{*}^{\prime} M^{\prime}\right) & =\left(f \times f^{\prime}\right)^{*}\left(p^{\prime *} f_{*} M \otimes q^{\prime *} f_{*}^{\prime} M^{\prime}\right) \\
& \simeq\left(\left(f \times f^{\prime}\right)^{*} p^{\prime *} f_{*} M\right) \otimes\left(\left(f \times f^{\prime}\right)^{*} q^{\prime *} f_{*}^{\prime} M^{\prime}\right) \\
& \simeq\left(p^{*} f^{*} f_{*} M\right) \otimes\left(q^{*} f^{\prime *} f_{*} M\right) \\
& \stackrel{a d j}{\longrightarrow} p^{*} M \otimes q^{*} M^{\prime}=M \otimes M^{\prime} .
\end{aligned}
$$

A typical example is given by $A(X):=D(X)$, the derived category of sheaves of $\mathcal{O}_{X}$-modules for $X$ a commutative ringed space, with $f^{*}=L f^{*}$ and $f_{*}=R f_{*}$ the derived inverse and direct image ([27][part I, (3.6.10) on p.124]). Or we can work with suitable subcategories, e.g. the subcategory $D_{q c}(X)$ of complexes with quasi-coherent cohomology in the context of $X$ a separated scheme. Here are some important examples of such "adjoint pairs":

(coh*) We are working with the category space of separated schemes of finite type over a base field $k$, with $A^{\prime}(X):=D_{q c}(X)$ and $f^{*}:=L f^{*}, f_{*}:=R f_{*}$ as before, see [27]. Note that the subcategory $A(X):=D_{c o h}^{b}(X)$ of bounded complexes with coherent cohomology is in general not stable under $f^{*}$ or $\otimes$. But it is stable under $f_{*}$ for proper morphisms, and under the exterior products $\otimes$, defining 
therefore a symmetric monoidal structure on $\left(D_{c o h}^{b}\right)^{o p} /$ space with space $:=s_{c h}^{c p}$ the category of separated complete schemes of finite type over a base field $k$.

$\left(c^{*}\right)$ We are working with the derived category $A(X):=D_{c}^{b}(X)$ of bounded complexes (of sheaves of vector spaces) with constructible cohomology in the complex algebraic (or analytic) context, with space the category of complex (quasi-projective) varieties (or the category of compact complex analytic spaces) [39]. Here we are using $\otimes$ and $f^{*}:=L f^{*}, f_{*}:=R f_{*}$ to get a symmetric monoidal structure on $\left(D_{c}^{b}\right)^{o p} /$ space.

The same arguments also work for constructible sheaf complexes in the context of real geometry for semialgebraic or compact subanalytic sets, and for stratified maps between suitable compact stratified sets [39].

$\left(\mathrm{MHM}^{*}\right)$ We are working with the derived category $A(X):=D^{b}(\operatorname{MHM}(X))$ of bounded complexes of mixed Hodge modules in the complex algebraic context [35, 37]. Here we are using Saito's functors $\otimes$ and $f^{*}, f_{*}$ to get a symmetric monoidal structure on $\left(D^{b}(\mathrm{MHM})\right)^{o p} /$ space.

Also note that in all these three examples the Künneth morphism Kue is an isomorphism (compare e.g. with [7][thm.2.1.2] for the case $\left(\mathrm{coh}^{*}\right)$, using the fact that a projection $X \times X^{\prime} \rightarrow X$ is a flat morphism, [39] [sec.1.4] for the case $\left(\mathrm{c}^{*}\right)$ and [35, 37] for the case $\left.\left(\mathrm{MHM}^{*}\right)\right)$ !

Example 4.3 (base change + projection morphism). This time the pseudofunctor $f_{*}:=$ $f_{\text {! }}$ is endowed with a natural projection morphism

$$
\left(f_{!} M\right) \otimes M^{\prime} \rightarrow f_{!}\left(M \otimes f^{*} M^{\prime}\right)
$$

and a natural base change morphism

$$
p^{*} f_{!} M \rightarrow\left(f \times i d_{X^{\prime}}\right) ! p^{*} M
$$

for $f, p, p^{\prime}$ as in the cartesion diagram (69) (with $X^{\prime}=Y^{\prime}$ ), satisfying suitable compabilities. Then one gets the Künneth morphism Kue for $f \times i d_{X^{\prime}}$ by

$$
\begin{aligned}
\left(f_{!} M\right) \otimes M^{\prime} & =\left(p^{*} f_{!} M\right) \otimes q^{*} M^{\prime} \\
& \rightarrow\left(\left(f \times i d_{X^{\prime}}\right) ! p^{*} M\right) \otimes q^{\prime *} M^{\prime} \\
& \rightarrow\left(f \times i d_{X^{\prime}}\right) !\left(p^{*} M \otimes\left(\left(f \times i d_{X^{\prime}}\right)^{*} q^{\prime *} M^{\prime}\right)\right. \\
& \simeq\left(f \times i d_{X^{\prime}}\right) !\left(p^{*} M \otimes q^{*} M^{\prime}\right) \\
& =\left(f \times i d_{X^{\prime}}\right) !\left(M \otimes M^{\prime}\right) .
\end{aligned}
$$

In the same way one gets the Künneth morphism for $i d_{X} \times f^{\prime}$ using the symmetry isomorphism s, and the Künneth morphism for $\left(f \times f^{\prime}\right)=\left(f \times i d_{X^{\prime}}\right) \circ\left(i d_{X} \times f^{\prime}\right)$ follows by composition.

For example, the structure of a "Green functor" in the sense of [16] for the pair of functors $(-)^{*},(-)$ ! is more than enough. The projection morphism comes from [16][(4.3)(ii)], 
whereas the base change morphism follows from [16][(3.3)(iii)].

Here are some important examples of such "pairs" $(-)^{*},(-)$ ! with projection and base change morphisms:

(c!) We are working with the derived category $A(X):=D_{c}^{b}(X)$ of bounded complexes (of sheaves of vector spaces) with constructible cohomology in the complex algebraic context, with space the category of complex (quasi-projective) varieties [39]. Here we are using $\otimes, f^{*}:=L f^{*}$ and the derived direct image functor with proper support $f_{!}:=R f_{!}$to get in this way a symmetric monoidal structure on $\left(D_{c}^{b}\right)^{o p} /$ space.

The same arguments also work for constructible sheaf complexes in the context of real geometry for semialgebraic sets [39].

(MHM!) We are working with the derived category $A(X):=D^{b}(\operatorname{MHM}(X))$ of bounded complexes of mixed Hodge modules in the complex algebraic context [35, 37]. Here we are using Saito's functors $\otimes, f^{*}$ and $f_{!}$to get in this way a symmetric monoidal structure on $\left(D^{b}(\mathrm{MHM})\right)^{o p} /$ space.

Also in these two examples, the projection and base change morphisms, and therefore also the Künneth morphisms Kue, are isomorphisms (compare e.g. with [39] [sec.1.4, cor.2.0.4] for the case (c!) and [35, 37] for the case (MHM!))!

Finally note, that there are also many interesting cases where one doesn't have such a "tensor structure" on $A(X)$ for a singular space $X$, e.g. for perverse sheaves, coherent sheaves or a compatible duality. But nevertheless $A^{o p} /$ space can be endowed with a symmetric monoidal structure as above so that our techniques and results apply.

\section{Appendix B: Mixed Hodge modules on symmetric products}

Let us recall the definition of symmetric products of a complex of mixed Hodge modules, where for the general background on the calculus of mixed Hodge modules we refer to [35, 36, 38]. Let $X$ be a complex quasi-projective variety, and denote by $X^{(n)}:=X^{n} / \Sigma_{n}$ its $n$-th symmetric product, with $p_{n}: X^{n} \rightarrow X^{(n)}$ the projection map. For $\mathcal{M} \in D^{b} \operatorname{MHM}(X)$, we let

$$
\mathcal{M}^{\bigotimes n} \in D^{b} \operatorname{MHM}\left(X^{n}\right)
$$

be the $n$-th external product of $\mathcal{M}$ with the induced $\Sigma_{n}$-action.

Remark 5.1. Let us point out that here we are regarding $\mathcal{M}^{\bigotimes n}$ as an equivariant object as defined in appendix A, and this corresponds to a "naive equivariant object" in the sense of [39][ch.3]. This is a weaker notion than that of an object in the equivariant derived category $D_{\Sigma_{n}}^{b} M H M\left(X^{n}\right)$ defined in the spirit of Bernstein-Lunts [5].

Since the projection map $p_{n}: X^{n} \rightarrow X^{(n)}$ is $\Sigma_{n}$-equivariant (with the trivial $\Sigma_{n}$-action on $\left.X^{(n)}\right)$, it follows that there is an induced $\Sigma_{n}$-action on

$$
p_{n_{*}} \mathcal{M}^{\otimes n} \in D^{b} \operatorname{MHM}\left(X^{(n)}\right) .
$$


Next we use the important fact from [[2], Cor. 2.10], that the bounded derived category $D^{b}(A)$ of an abelian category $A$ is an idempotent complete (or Karoubian) additive category, that is, any projector

$$
\mathcal{P}=\mathcal{P}^{2}: F \rightarrow F \quad \text { on an object } \quad F \in D^{b}(A)
$$

arises from a splitting

$$
F \simeq K \oplus I, \quad \text { with }\left.\mathcal{P}\right|_{K}=0 \text { and }\left.\mathcal{P}\right|_{I}=i d .
$$

Call $K=\operatorname{Ker}(\mathcal{P})$ the kernel and $I=\mathcal{P}(F)$ the image of $\mathcal{P}$. If we now let $A=$ $\operatorname{MHM}\left(X^{(n)}\right)$, the abelian category of Saito's algebraic mixed Hodge modules on $X^{(n)}$, it follows by this result that $D^{b} \operatorname{MHM}\left(X^{(n)}\right)$ is an idempotent complete additive category. Moreover, $D^{b} \operatorname{MHM}\left(X^{(n)}\right)$ is also a $\mathbb{Q}$-linear category (i.e., the Hom's in the category are $\mathbb{Q}$-vector spaces). So the functor

$$
\mathcal{P}:=\frac{1}{n !} \sum_{\sigma \in \Sigma_{n}} \psi_{\sigma}
$$

is a well-defined projector on the category of $\Sigma_{n}$-equivariant objects in $D^{b} \operatorname{MHM}\left(X^{(n)}\right)$ in the sense of appendix A, with $\psi_{\sigma}$ the isomorphisms coming from the $\Sigma_{n}$-action. Finally, for $\mathcal{M} \in D^{b} \operatorname{MHM}(X)$, we let

$$
\mathcal{M}^{(n)}:=\mathcal{P}\left(p_{n_{*}} \mathcal{M}^{\bigotimes n}\right) \in D^{b} \operatorname{MHM}\left(X^{(n)}\right)
$$

be the $n$-th symmetric product of $\mathcal{M}$.

Remark 5.2. The same construction works for the derived categories $D_{c}^{b}(X)$ and $D_{\text {coh }}^{b}(X)$ needed in Theorem 1.4, because these are $\mathbb{Q}$-linear triangulated categories with a bounded $t$-structure, so they are also Karoubian by [26].

Next we explain some important examples of such symmetric products of (complexes of) mixed Hodge modules. First we assume that $\mathcal{M}=\mathbb{Q}_{X}^{H}:=k^{*} \mathbb{Q}_{p t}^{H}$ is the constant Hodge module, for $k: X \rightarrow p t$ the constant map to a point, and where

$$
\mathbb{Q}_{p t}^{H} \in \mathrm{MHM}(\text { point }) \simeq \mathrm{mHs}^{p}
$$

is identified with the mixed Hodge structure $\mathbb{Q}$ of type $(0,0)$. The following result supplies a proof of equation (14):

Lemma 5.3. For a complex quasi-projective variety $X$ we have that

$$
\left(\mathbb{Q}_{X}^{H}\right)^{(n)} \simeq \mathbb{Q}_{X^{(n)}}^{H} \in D^{b} \operatorname{MHM}\left(X^{(n)}\right) .
$$

Proof. Consider the composite morphism

$$
\mathbb{Q}_{X}^{H} \rightarrow p_{n_{*}}\left(\mathbb{Q}_{X}^{H}\right)^{\otimes n} \rightarrow\left(\mathbb{Q}_{X}^{H}\right)^{(n)},
$$

where the first arrow is induced by the adjunction morphism

$$
\text { id } \rightarrow p_{n *} p_{n}^{*}
$$

together with the identification $p_{n}{ }^{*} \mathbb{Q}_{X(n)}^{H}=\mathbb{Q}_{X^{n}}^{H} \cong\left(\mathbb{Q}_{X}^{H}\right)^{\bigotimes n}$, and the second is induced by the symmetric projector $\mathcal{P}$ above. The claim is that the morphism (74) is 
in fact an isomorphism. Notice that this is equivalent to showing that the cone of (74) is $0 \in D^{b} \operatorname{MHM}(Z)$. And this can be checked after applying the forgetful functor rat : $D^{b} \operatorname{MHM}\left(X^{(n)}\right) \rightarrow D_{c}^{b}\left(X^{(n)}\right)$. More precisely, one has the following sequence of equivalences for a mixed Hodge module complex on an complex algebraic variety $Z$ :

$$
\begin{aligned}
\mathcal{M} \simeq 0 \in D^{b} \operatorname{MHM}(Z) & \Leftrightarrow H^{i}(\mathcal{M}) \simeq 0 \in \operatorname{MHM}(Z), \text { for all } i \\
& \Leftrightarrow \operatorname{rat}\left(H^{i}(\mathcal{M})\right)={ }^{p} \mathcal{H}^{i}(\operatorname{rat}(\mathcal{M})) \simeq 0 \in \operatorname{Perv}(Z), \text { for all } i \\
& \Leftrightarrow \operatorname{rat}(\mathcal{M}) \simeq 0 \in D_{c}^{b}(Z) .
\end{aligned}
$$

Here the first equivalence is by the definition of a derived category, the second uses the faithfulness of the forgetful functor rat : $\operatorname{MHM}(Z) \rightarrow \operatorname{Perv}(Z)$ associating to a mixed Hodge module the underlying perverse sheaf (with ${ }^{p} \mathcal{H}^{*}(-$ ) denoting the perverse cohomology groups), and the last one follows from the boundedness of the perverse $t$ structure on $D_{c}^{b}(Z)$. Lastly, the underlying map $\mathbb{Q}_{X}^{(n)} \rightarrow\left(\mathbb{Q}_{X}\right)^{(n)}$ is an isomorphism by [21] [ch.V].

Let us now assume that $X$ is pure-dimensional, and consider the case

$$
\mathcal{M}=I C_{X}^{\prime H}:=I C_{X}^{H}\left[-\operatorname{dim}_{\mathbb{C}} X\right] .
$$

Lemma 5.4. If $X$ is a pure-dimensional complex quasi-projective variety, the following isomorphism holds:

$$
\left(I C_{X}^{\prime H}\right)^{(n)} \simeq I C_{X^{(n)}}^{H} \in D^{b} M H M\left(X^{(n)}\right) .
$$

Proof. Let $U \subset X$ be a smooth Zariski-open dense subset, which is assumed to be affine. Then if $j: U \hookrightarrow X$ denotes the inclusion map, the functors

$$
j_{*}, j_{!}: \operatorname{MHM}(U) \rightarrow \operatorname{MHM}(X)
$$

are exact. It follows that the same is true for the induced inclusion $j^{n}: U^{n} \hookrightarrow X^{n}$, where for $\mathcal{M} \in D^{b} \operatorname{MHM}(U)$ we have that

$$
\left(j^{n}\right) !\left(\mathcal{M}^{\bigotimes n}\right)=(j ! \mathcal{M})^{\bigotimes n}
$$

and

$$
\left(j^{n}\right)_{*}\left(\mathcal{M}^{\otimes n}\right)=\left(j_{*} \mathcal{M}\right)^{\bigotimes n} .
$$

Denote by $j^{\prime}: U^{(n)} \hookrightarrow X^{(n)}$ the induced open embedding, and by $p_{n}^{\prime}$ the restriction of $p_{n}$ to $U^{n}$. These satisfy the relation $p_{n} \circ j^{n}=j^{\prime} \circ p_{n}^{\prime}$. We also let the symbol "! *" stand for the intermediate extension for an open embedding, which is formally defined by the rule: "!* $=$ image $(! \rightarrow *)$ ". Then the following identity holds for any $\mathcal{M} \in \operatorname{MHM}(U)$ :

$$
j_{! *}^{\prime}\left(p_{n *}^{\prime} \mathcal{M}^{\otimes n}\right)=p_{n *}\left(j^{n}\right)_{! *} \mathcal{M}^{\bigotimes n} .
$$

Indeed, we start by considering the defining sequence for the intermediate extension $\left(j^{n}\right)_{! *} \mathcal{M}^{\bigotimes n}$, i.e., the sequence

$$
\left(j^{n}\right) ! \mathcal{M}^{\bigotimes n} \rightarrow\left(j^{n}\right)_{!_{*}} \mathcal{M}^{\bigotimes n} \hookrightarrow\left(j^{n}\right)_{*} \mathcal{M}^{\bigotimes n}
$$


in the abelian category $\operatorname{MHM}\left(X^{n}\right)$. By applying the exact functor $p_{n !}=p_{n_{*}}$ for the finite (hence proper) map $p_{n}: X^{n} \rightarrow X^{(n)}$ to the sequence of (79), we obtain the sequence

$$
p_{n !}\left(j^{n}\right)_{!} \mathcal{M}^{\bigotimes n} \rightarrow p_{n_{*}}\left(j^{n}\right)_{!_{*}} \mathcal{M}^{\bigotimes n} \hookrightarrow p_{n_{*}}\left(j^{n}\right)_{*} \mathcal{M}^{\bigotimes n}
$$

in the abelian category $\operatorname{MHM}\left(X^{(n)}\right)$. Lastly, using the identities $p_{n *}\left(j^{n}\right)_{*}=j_{*}^{\prime} p_{n *}^{\prime}$ and $p_{n !}\left(j^{n}\right)_{!}=j_{!}^{\prime} p_{n !}^{\prime}$, with $p_{n !}^{\prime}=p_{n *}^{\prime}$ for the finite map $p_{n}^{\prime}$, we easily obtain (78).

Since in the abelian category $\operatorname{MHM}(-)$ the projector $\mathcal{P}$ of $(72)$ is also exact in short exact sequences of $\Sigma_{n}$-equivariant mixed Hodge modules, it commutes with the functor $j_{!_{*}}^{\prime}$, and it follows that:

$$
\begin{aligned}
j_{!_{*}}^{\prime}\left(\mathcal{M}^{(n)}\right) & =j_{!_{*}}^{\prime}\left(\mathcal{P}\left(p_{n *}^{\prime} \mathcal{M}^{\nabla n}\right)\right) \\
& \simeq \mathcal{P}\left(j_{!_{*}}^{\prime}\left(p_{n *}^{\prime} \mathcal{M}^{\nabla n}\right)\right) \\
& \stackrel{\sqrt{78}}{\simeq} \mathcal{P}\left(p_{n *}\left(j^{n}\right)_{!_{*}} \mathcal{M}^{\bigotimes n}\right) \\
& \simeq \mathcal{P}\left(p_{n *}\left(j_{!_{*}} \mathcal{M}\right)^{\bigotimes n}\right) \\
& =\left(j_{!_{*}} \mathcal{M}\right)^{(n)} .
\end{aligned}
$$

Finally, for $\mathcal{M}=\mathbb{Q}_{U}^{H}$ and by using Lemma 5.3, we obtain:

$$
\begin{aligned}
\left(I C^{\prime H}{ }^{H}\right. & (n) \\
& \stackrel{\text { def }}{=}\left(j_{!_{*}} \mathbb{Q}_{U}^{H}\right)^{(n)} \\
& \simeq j_{!_{*}}^{\prime}\left(\left(\mathbb{Q}_{U}^{H}\right)^{(n)}\right) \\
& \simeq j_{!_{*}}^{\prime}\left(\mathbb{Q}_{U^{(n)}}^{H}\right) \\
& =I C_{X^{(n)}}^{H} .
\end{aligned}
$$

The arguments used in the proof of Lemma 5.4 can also be adapted in order to describe the complex $\left(I C^{\prime H}(\mathcal{L})\right)^{(n)} \in D^{b} \operatorname{MHM}\left(X^{(n)}\right)$, for $\mathcal{L}$ an admissible variation of mixed Hodge structures defined on a smooth Zariski-open dense subset $U$ of $X$. Note that even if $U^{n}$ is smooth, $U^{(n)}$ is singular, and extra care is needed in order to make sense of $\mathcal{L}^{(n)}$, where we also denote by $\mathcal{L}$ the complex of mixed Hodge modules in $D^{b} \mathrm{MHM}(U)$ corresponding to the admissible variation (cf. [35]). However, in the notations of Lemma 5.4, the finite projection $p_{n}^{\prime}: U^{n} \rightarrow U^{(n)}$ is a small map, so that outside the "fat diagonal", i.e., on the configuration space on $n$ ordered points

$$
F(U, n):=\left\{\left(x_{1}, x_{2}, \cdots, x_{n}\right) \in U^{n} \mid x_{i} \neq x_{j} \text { for } i \neq j\right\}
$$

it restricts to a finite covering map $p_{n}^{\prime \prime}: F(U, n) \rightarrow B(U, n):=F(U, n) / \Sigma_{n}$ of complex manifolds. It then follows that $p_{n *}^{\prime \prime}\left(\left.\mathcal{L}^{\bigotimes n}\right|_{F(U, n)}\right)$ is an admissible variation of Hodge structures on $B(U, n)$ and we can define as before:

$$
\mathcal{L}^{(n)}:=\mathcal{P}\left(p_{n *}^{\prime \prime}\left(\left.\mathcal{L}^{\otimes n}\right|_{F(U, n)}\right)\right) .
$$

Lemma 5.5. With the above notations, the following isomorphism holds

$$
\left(I C^{\prime H}(\mathcal{L})\right)^{(n)} \simeq I C_{X}^{\prime H}\left(\mathcal{L}^{(n)}\right) \text {. }
$$


Proof. Since $p_{n}^{\prime}$ is a small map, by base change we obtain that

$$
p_{n *}^{\prime}\left(\mathcal{L}^{\bigotimes n}\right)=I C_{U^{(n)}}^{\prime H}\left(p_{n *}^{\prime \prime}\left(\left.\mathcal{L}^{\bigotimes n}\right|_{F(U, n)}\right)\right) .
$$

Let $i^{\prime}: B(U, n) \hookrightarrow U^{(n)}$ denote the open inclusion. Then it follows just like in the proof of Lemma 5.4 that

$$
\begin{aligned}
& \left(I C^{\prime H}(\mathcal{L})\right)^{(n)} \stackrel{\text { def }}{=}\left(j_{! *} \mathcal{L}\right)^{(n)} \\
& =\mathcal{P}\left(p_{n_{*}}\left(j_{!_{*}} \mathcal{L}\right)^{\bigotimes n}\right) \\
& \simeq \mathcal{P}\left(p_{n *}\left(j^{n}\right)_{! *} \mathcal{L}^{\bigotimes n}\right)
\end{aligned}
$$

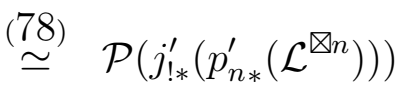

$$
\begin{aligned}
& \simeq \mathcal{P}\left(\left(j^{\prime} \circ i^{\prime}\right)_{! *}\left(p_{n *}^{\prime \prime}\left(\left.\mathcal{L}^{\otimes n}\right|_{F(U, n)}\right)\right)\right) \\
& =\left(j^{\prime} \circ i^{\prime}\right)_{! *}\left(\mathcal{L}^{(n)}\right) \\
& =I C^{\prime H}{ }^{(n)}\left(\mathcal{L}^{(n)}\right) \text {. }
\end{aligned}
$$

Remark 5.6. Using the alternating projector, a similar proof can be given for the isomorphism (28) from the introduction. This follows from the following equality of variations of mixed Hodge structures on the configuration space $B(U, n)$ :

$$
\mathcal{L}^{\{n\}}:=\left(p_{n *}^{\prime \prime}\left(\left.\mathcal{L}^{\otimes n}\right|_{F(U, n)}\right)\right)^{s i g n-\Sigma_{n}} \simeq\left(\epsilon_{n} \otimes p_{n *}^{\prime \prime}\left(\left.\mathcal{L}^{\otimes n}\right|_{F(U, n)}\right)\right)^{\Sigma_{n}} \simeq \epsilon_{n} \otimes \mathcal{L}^{(n)} .
$$

Lemma 5.7. Let $j: B(X, n) \rightarrow X^{(n)}$ be the open inclusion of the configuration space $B(X, n)$ of all unordered $n$-tuples of different points in the complex quasi-projective variety $X$, with $i$ the closed inclusion of the complement of $B(X, n)$ into $X^{(n)}$. Then for $\mathcal{M} \in D^{b} \operatorname{MHM}(X)$ the following formulae hold:

$$
j ! j^{*} \mathcal{M}^{\{n\}} \simeq \mathcal{M}^{\{n\}} \text { and } i^{*} \mathcal{M}^{\{n\}} \simeq 0 .
$$

Proof. Recall that the alternating object $\mathcal{M}^{\{n\}}$ is defined as

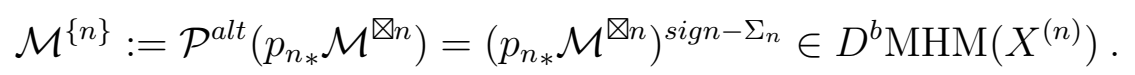

It is enough to prove the isomorphism $i^{*} \mathcal{M}^{\{n\}} \simeq 0$ since by Saito's work ([35]) there is a distinguished triangle in $D^{b} \operatorname{MHM}\left(X^{(n)}\right)$ :

$$
j ! j^{*} \mathcal{M}^{\{n\}} \longrightarrow \mathcal{M}^{\{n\}} \longrightarrow i_{*} i^{*} \mathcal{M}^{\{n\}} \stackrel{[1]}{\longrightarrow} .
$$

As in (75), in order to show the isomorphism $i^{*} \mathcal{M}^{\{n\}} \simeq 0$, it is sufficient to check it on the underlying constructible complex

$$
\mathcal{F}^{\{n\}}=\operatorname{rat}\left(\mathcal{M}^{\{n\}}\right) \simeq \mathcal{P}^{a l t}\left(p_{n_{*}} \mathcal{F}^{\bigotimes n}\right)=\left(p_{n_{*}} \mathcal{F}^{\bigotimes n}\right)^{\text {sign- } \Sigma_{n}}
$$

with $\mathcal{F}:=\operatorname{rat}(\mathcal{M})$. And for this it is enough to show the vanishing of its stalk cohomology $\mathbb{Q}$-vector spaces, i.e., $H^{k}\left(\mathcal{F}_{\bar{x}}^{\{n\}}\right)=0$ for all $k \in \mathbb{Z}$ and all $\bar{x}=\left(x_{1}, \ldots, x_{n}\right) \in$ $X^{(n)} \backslash B(X, n)$. Since the projection $p_{n}: X^{n} \rightarrow X^{(n)}$ is finite, $p_{n *}$ is exact and one has

$$
H^{k}\left(\left(p_{n_{*}} \mathcal{F}^{\bigotimes n}\right)_{\bar{x}}\right) \simeq \oplus_{\left\{\bar{y} \mid p_{n}(\bar{y})=\bar{x}\right\}} H^{k}\left(\mathcal{F}_{\bar{y}}^{\bigotimes n}\right) .
$$


And the stalk cohomology $H^{k}\left(\mathcal{F}_{\bar{x}}^{\{n\}}\right)$ is just a direct summand of $H^{k}\left(\left(p_{n *} \mathcal{F}^{\bigotimes n}\right)_{\bar{x}}\right)$ defined by the alternating projector. So it suffices to show that for each $\bar{y} \in p_{n}^{-1}(\{\bar{x}\})$,

$$
0=H^{k}\left(\mathcal{F}_{\bar{x}}^{\{n\}}\right) \cap H^{k}\left(\mathcal{F}_{\bar{y}}^{\bigotimes n}\right) \subset H^{k}\left(\left(p_{n *} \mathcal{F}^{\bigotimes n}\right)_{\bar{x}}\right) .
$$

Since $\bar{x}=\left(x_{1}, \ldots, x_{n}\right) \in X^{(n)} \backslash B(X, n)$, there is for each $\bar{y} \in p_{n}^{-1}(\{\bar{x}\})$ a transposition $\tau \in \Sigma_{n}$ fixing $\bar{y}$. This implies that $H^{k}\left(\mathcal{F}_{\bar{y}}^{\bigotimes n}\right)$ is invariant under the symmetry isomorphism $H^{k}\left(\psi_{\tau}\right)$ coming from the $\Sigma_{n}$-action. On the other hand, by the definition of the alternating projector, $H^{k}\left(\mathcal{F}_{\bar{x}}^{\{n\}}\right)$ is invariant under the isomorphism $-H^{k}\left(\psi_{\tau}\right)$. Since these are $\mathbb{Q}$-vector spaces, there is no 2-torsion and the above facts imply the vanishing of $H^{k}\left(\mathcal{F}_{\bar{x}}^{\{n\}}\right) \cap H^{k}\left(\mathcal{F}_{\bar{y}}^{\bigotimes n}\right)$.

\section{REFERENCES}

[1] M. F. Atiyah, Power operations in K-theory, Quart. J. Math. 17 (1966), 165-193.

[2] P. Balmer, M. Schlichting, Idempotent completion of triangulated categories, Journal of Algebra 236 (2001), 819-834.

[3] M. Banagl, S. E. Cappell, J. L. Shaneson, Computing twisted signatures and L-classes of stratified spaces, Math. Ann. 326 (2003), 589-623.

[4] P. Baum, W. Fulton, R. MacPherson, Riemann-Roch for singular varieties, Publ. Math. I.H.E.S. 45, 101-145 (1975).

[5] J. Bernstein, V. Lunts, Equivariant sheaves and functors, LNM 1578.

[6] S. Biglari, A Künneth formula in tensor triangulated categories, Journal of Pure and Applied Algebra 210 (2007), 645-650.

[7] J. Bonsdorff, A Fourier transformation for Higgs bundles, J. Reine Angew. Math. 591 (2006), 21-48.

[8] F. Borceux, Handbook of Categorical Algebra 2, Encyclopedia of Mathematics and its Applications 51, Combridge University Press, 1994.

[9] L. Borisov, A. Libgober, Elliptic genera of singular varieties, orbifold elliptic genus and chiral de Rham complex, in Mirror symmetry, IV (Montreal, QC, 2000), 325-342, AMS/IP Stud. Adv. Math., 33, Amer. Math. Soc., Providence, RI, 2002.

[10] J. P. Brasselet, J. Schürmann, S. Yokura, Hirzebruch classes and motivic Chern classes of singular spaces, arXiv:math/0503492.

[11] S. E. Cappell, L. Maxim, J. L. Shaneson, Equivariant genera of complex algebraic varieties, Int. Math. Res. Notices 2009 (2009), 2013-2037.

[12] J. Cheah, On the cohomology of Hilbert schemes of points, J. Algebraic Geometry 5 (1996), 479-511.

[13] P. Deligne, Théorie de Hodge, II, III, Publ. Math. IHES 40, 44 (1972, 1974).

[14] P. Deligne, Catégories tensorielles, Moscow Math. Journal 2 (2002), 227-248.

[15] E. Getzler, Mixed Hodge structures on configuration spaces, arXiv:math/9510018.

[16] E. Getzler, Resolving mixed Hodge modules on configuration spaces, Duke Math. J. 96 (1999), no. 1, 175-203.

[17] J. M. Gómez, From fibered symmetric bimonoidal categories to symmetric spectra, arXiv:math/0905.3156.

[18] M. Goresky, R. MacPherson, Intersection Homology, Topology 19 (1980), 135-162.

[19] E. Gorsky, Adams operations and power structures, arXiv:math/0803.3118.

[20] L. Göttsche, W. Soergel, Perverse sheaves and the cohomology of Hilbert schemes of smooth algebraic surfaces, Math. Ann. 296 (1993), 235-245. 
[21] A. Grothendieck, Sur quelques points d'algèbre homologique. Tôhoku Math. J. (2) 9 1957, 119-221.

[22] T. Hausel, F. Rodriguez-Villegas, Mixed Hodge polynomials of character varieties, Inv. Math. 174 (2008), 555-624.

[23] F. Heinloth, A note on functional equations for zeta functions with values in Chow motives, Annales de L'Inst. Fourier 57 (2007), 1927-1945.

[24] M. Kapranov, The elliptic curve in S-duality theory and Eisnestein series for Kac-Moody groups, arXiv:math/0001005.

[25] D. Knutson, $\lambda$-Rings and the Representation Theory of the Symmetric Group, Lecture Notes in Mathematics, Vol. 308. Springer-Verlag, Berlin-New York, 1973.

[26] J. Le, X.-W. Chen, Karoubianness of a triangulated category, Journal of Algebra 310 (2007), 452457.

[27] J. Lipmann, M. Hashimoto, Foundations of Grothendieck Duality for Diagrams of Schemes, Lecture Notes in Mathematics, Vol. 1960. Springer-Verlag, Berlin-New York, 2009.

[28] I. G. Macdonald, Symmetric products of an algebraic curve, Topology 1 (1962), 319-343.

[29] I. G. Macdonald, The Poincaré polynomial of a symmetric product, Proc. Cambridge Philos. Soc. 58, 1962, 563 - 568.

[30] R. MacPherson, Chern classes for singular algebraic varieties, Ann. of Math. (2) 100 (1974), 423432.

[31] S. McGarraghy, Exterior powers of symmetric bilinear forms, Algebra Colloq. 9 (2002), no. 2, 197-218.

[32] S. McGarraghy, Symmetric powers of symmetric bilinear forms, Algebra Colloq. 12 (2005), no. 1, $41-57$.

[33] B. Moonen, Das Lefschetz-Riemann-Roch-Theorem für singuläre Varietäten, Bonner Mathematische Schriften 106 (1978), viii+223 pp.

[34] T. Ohmoto, Generating functions for generating Chern classes I: symmetric products, Math. Proc. Cambridge Philos. Soc. 144 (2008), no. 2, 423-438.

[35] M. Saito, Mixed Hodge Modules, Publ. Res. Inst. Math. Sci. 26 (1990), no. 2, 221-333.

[36] M. Saito, Introduction to mixed Hodge modules, Actes du Colloque de Théorie de Hodge (Luminy, 1987), Astérisque No. 179-180 (1989), 10, 145-162.

[37] M. Saito, On the formalism of mixed sheaves, arXiv:math/0611597.

[38] M. Saito, Mixed Hodge complexes on algebraic varieties, Math. Ann. 316 (2000), 283-331.

[39] J. Schürmann, Topology of singular spaces and constructible sheaves, Monografie Matematyczne 63 (New Series), Birkhäuser, Basel, 2003.

[40] Sém. géométrie algébrique (1967-1969). Théorie des topos et cohomologie étale. Part III, Lecture Notes in Mathematics, Vol. 305. Springer-Verlag, Berlin-New York, 1973.

[41] A. Vistoli, Grothendieck topologies, fibered categories and decent theory, Math. Surveys Monogr. 123 (2005), 1-104.

[42] D. Zagier, Equivariant Pontrjagin classes and applications to orbit spaces. Applications of the Gsignature theorem to transformation groups, symmetric products and number theory, Lecture Notes in Mathematics, Vol. 290. Springer-Verlag, Berlin-New York, 1972.

[43] J. Zhou, Calculations of the Hirzebruch $\chi_{y}$ genera of symmetric products by the holomorphic Lefschetz formula, arXiv:math/9910029.

L. Maxim : Courant Institute, New York University, 251 Mercer Street, New York CITY, NY 10012

E-mail address: maxim@cims.nyu.edu

J. Schürmann : Mathematische Institut, Universität MÜnster, Einsteinstr. 62, 48149 Münster, Germany.

E-mail address: jschuerm@math.uni-muenster.de 